\title{
PREOPERATIVE SURGICAL PLANNING FOR ROBOT-ASSISTED LIVER TUMOUR ABLATION THERAPY BASED ON COLLISION-FREE REACHABLE WORKSPACES
}

\author{
Shaoli Liu, ${ }^{*}$ Jianhua Liu, ${ }^{*}$ Jing $\mathrm{Xu},{ }^{* *}$ Xiaoyu Ding, ${ }^{*}$ Tong Lu, ${ }^{* * *}$ and Ken Chen ${ }^{* *}$
}

\begin{abstract}
To destroy an entire tumour with the minimum damage to healthy tissue using robotic-assisted liver tumour coagulation therapy, the surgeon should manually determine the needle-insertion path and the entry point based on a reconstructed three-dimensional (3D) model of the liver and the operating environment. However, existing preoperative planning methods depend on the skill of the surgeon, which has implications for the surgeon's workload. Because of a lack of tactile feedback and limited 3D visualization space, this preoperative planning must be iterated many times until an optimal needle-insertion path can be obtained. Here we propose a preoperative planning method that determines the collision-free reachable workspace (CFRW) of the needle, from which the surgeon can select a feasible needle-insertion path, or the optimal needleinsertion path can be automatically chosen with some optimization criteria. Based on a mathematical description of the critical structures, an analytical expression of the CFRW of the needle is obtained, whereby the needle-insertion path can avoid interference with blood vessels, ribs and other obstacles. This work provides an effective and accurate method to aid the surgeon in selecting a needle-insertion path, which can improve the efficiency and safety of the operation, as well as significantly reduce the surgeon's workload.
\end{abstract}

\section{Key Words}

Boundary of collision-free reachable workspace, trajectory planning, robotic-assisted surgery, ablation therapy

* School of Mechanical Engineering, Beijing Institute of Technology, People's Republic of China, Beijing; e-mail: \{liushaoli, jeffliu, xiaoyu.ding\}@bit.edu.cn

** Department of Mechanical Engineering, Tsinghua University, People's Republic of China, Beijing; e-mail: \{jingxu, kenchen\}@tsinghua.edu.cn

*** Department of Ultrasound, The Chinese PLA General Hospital, People's Republic of China, Beijing; e-mail: lutong@fitme.org

Recommended by Dr. Peter Liu

(DOI: 10.2316/Journal.206.2017.5.206-4607)

\section{Introduction}

Over the past decade, ablation therapy, which is minimally invasive surgery, has become widely used in clinical treatment of malignant liver tumours [1], due to the benefits in pain relief for patients, avoiding infection, reducing the recovery time and overall low cost. Recently, dexterous robot systems have been introduced, including the 3D ultrasound (US) image-guided robotic system developed by our group for improved microwave (MW) ablation of malignant liver tumours [2]. Typical operation procedures using this robotic system consist of three steps: preoperative surgical planning, intraoperative therapy and postoperative assessment. Figure 1 shows a diagram of operation of the robotic-assisted liver tumour coagulation therapy (RALTCT) system. During the preoperative step, the surgeon carried out surgical planning to identify a feasible needle-insertion path and the corresponding entry point with the help of a 3D liver tumour model reconstructed using a free-hand tracked US imaging module. In the intraoperative step, the robot then accurately places the needle into the target according to the planned path. Intensive experiments have been carried out, as reported by us previously, which indicate that a significant improvement of the needle placement accuracy could be achieved using the robot-assisted system [2]. Therefore, with a suitable preoperative planning strategy, the robot system is expected to enable complete destruction of malignant tumours with minimal damage to healthy tissue.

Of these three operating steps, the preoperative planning is the most important, as it affects all of the subsequent steps and thus determines the overall treatment effectiveness. The primary objective of preoperative planning is to identify the optimal needle-insertion path to the target without damaging important organs or tissues (e.g., ribs or primary blood vessels). However, with manual preoperative planning, the experience and skill of the surgeon significantly affects the chances of complete ablation, 


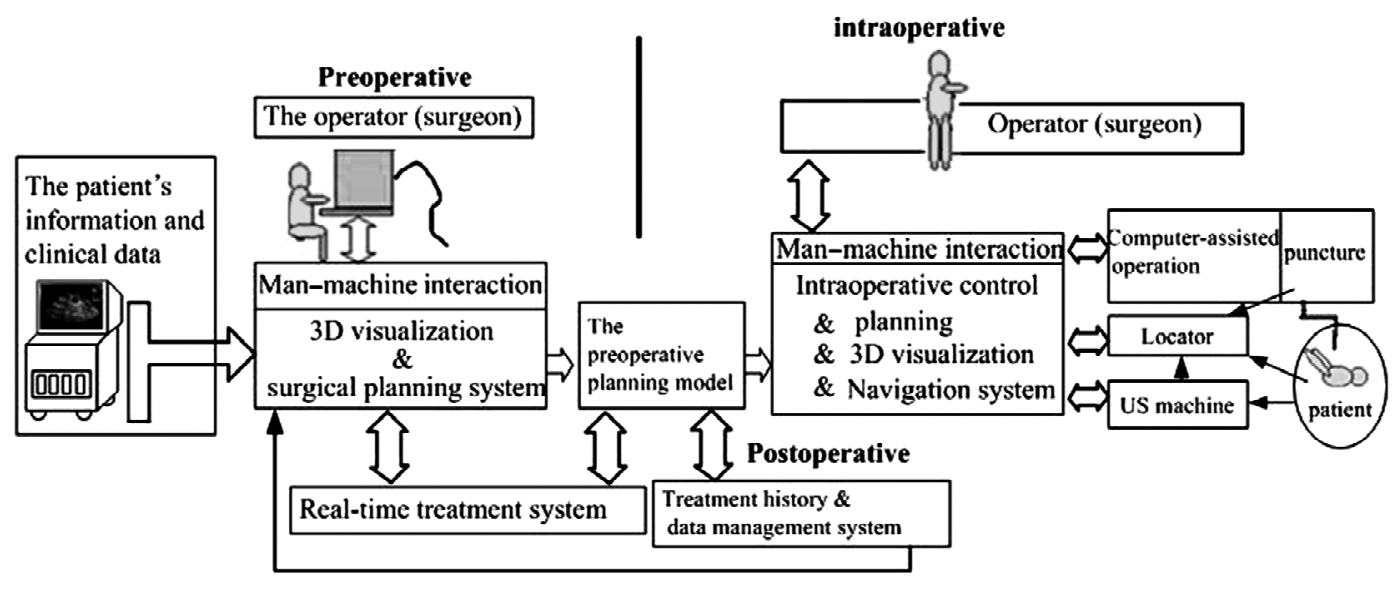

Figure 1. A diagram of the operation procedure of the RALTCT system.

as well as the risks of complications. Moreover, the selection of an appropriate path must be repeated several times until a feasible needle-insertion path can be obtained, during which many clinical constrains and the constraints of the movement of the robot should be taken into account by the surgeon manually. For this reason, the existing preoperative planning method requires improvement to reduce the surgeon's workload and enhance the planning efficiency and quality.

\section{Related Work}

In the following, we review related planning methods for robot-assisted surgery. These methods can be classified into: computer-assisted manual planning (CAMP), semiautonomous planning (SAP) and autonomous planning (AP), according to the level of manual interaction during planning.

Of these three methods, CAMP requires the highest level of manual interaction. For example, Peterhans et al. [3] developed a 3D surgical planning navigation platform that is suitable for clinical laparotomy of liver cancer. The navigation platform provides an effective $3 \mathrm{D}$ model of the liver, rapid intraoperative registration and precise tracking for surgical instruments. The navigation platform has already successfully assisted surgeons in carrying out nine surgical operations. Using the analysis of computed tomography (CT) image data, Shevchenko et al. [4] reconstructed the 3D shape of blood vessels and the tumour inside the liver using automatic and semi-automatic segmentation techniques. The distance between the tumour and major blood vessels was obtained via risk analysis [5], and the blood vessels that were most at greatest risk of damage were identified. This information can be used to assist the surgeon in preoperative planning. Chen et al. [6] reported a computer-aided liver surgical planning system composed of three modules: liver segmentation, vessel extraction, and visualization and interaction to prepare a virtual environment for surgical planning. Zhong et al. [7] proposed a computer-based surgical simulation to track the position of the surgical instrument and render force feedback to the user. Banz et al. [8] provided an analysis and assessment of suitable indications for image guidance in the domain of open liver surgery. The surgical navigation system has a sterile, transparent drap, which is used to interact with surgical team, and an optical tracking camera to monitor any interference between the tracking line and other objects. The surgical instruments and the US probe were attached with geometric instrument calibration for spatial tracking. Abdullah et al. [9] reported a Maxio workstation for simulation and treatment planning. The application software allowed $2 \mathrm{D}$ and $3 \mathrm{D}$ visualization to help the operator select the entry point of insertion manually. Then, the operator ensures or modifies the insertion trajectory by manually scrolling the image to determine whether the needle trajectory interfere with any critical structures. Scherer et al. [10] present a navigation system that consists of a display system, a set of tracked devices used for spatial localization and a localizing system to track these instruments in surgical space. The surgeons can interact with the system through the touch screens. Chebbi et al. [11] present a collaborative hapto-visual virtual system for simulating a surgery environment and graphical rendering. It can assist users to perform simple tasks and evaluate their performance by sensing their movements through the haptic device. Cristina et al. [12] reported a system called VirSSPA, which generates personalized biomodels of patients in virtual reality, using techniques including image analysis, segmentation and modelling. The system can support interventions and training of surgeons. The 3D Slicer [13] and the NeuroMate ${ }^{\mathrm{TM}}$ neurosurgical robot [14] also use such CAMP methods. With the assistance of the surgical navigation software, these systems allow the surgeon to interactively select key points (i.e., entry and target points) for needle insertion and to assess the surgical plan via simulations. Moreover, based on a criterion such as the needle-insertion length, which is calculated by the software, the display of the medical tools and the view of the important tissues close to the medical tools, the surgeon can adjust the surgical plan to identify the optimal needle-insertion path or amend a plan. CAMP is a relatively low-efficiency technique, and the plans generated using it still depend on the surgeon's experience. In summary, CAMP is suitable for a relatively simple 
surgery, such as neurosurgery or single-needle insertion in abdominal surgery.

SAP requires an intermediate level of manual interaction. With this method, explicit planning principles, which can be translated to mathematical models, are handled by the computer and/or the medical robot. Empirical factors and uncertainties during the planning process are handled by the surgeon. With computer-assisted SAP, the robot can adjust its path according to certain control strategies and/or affect the manual input via certain feedback mechanisms; e.g., haptic/tactile feedback [15]. The concept of a "virtual fixture" (VF) [16]-[18] is commonly used in SAP. Park et al. [19] reported a planning method by establishing a VF-based "virtual wall". This method may prevent medical tools from entering certain restricted areas when using the Zeus surgical robot for cardiac surgery. Kapoor et al. [20] reported a VF-based task primitive library such that the surgical operation is transformed into a constrained optimization problem, which is used to assist in surgical planning. Duan et al. [21] present a semi-automatic planning method to decide the placement of the radiofrequency ablation (RFA) needle. A cost function is defined, where the number of needle, number of voxels in each spherical destruction and the target are all considered. Then, the downhill simplex optimization is used to find the optimal needle placement. However, lots of constraints in real surgery were not considered, such as the insertion path cannot be too close to the vital tissues, the shortest insertion path and so on. Ren et al. [22] developed a semi-automatic planning and guidance method for multiple overlapping RFAs. Multiple-objective optimization, including both clinical and technical constraints, was used. However, at the beginning, the clinician must specify a set of entry points manually. Thus, the optimal results are local optima. This method can be used for both preoperative and intraoperative planning, because the algorithm can be implemented in real time. One problem with these method, however, is that it has many empirical coefficients (the values must be identified experimentally), which limits its further application.

AP is an efficient preoperative planning method that requires very few manual interactions. It is based on $3 \mathrm{D}$ imaging information and parameterized empirical models, and an appropriate surgical plan can be obtained using optimization methods. The planning results mainly depend on empirical models, rather than the surgeon's experience. For this reason, AP is commonly used in robotassisted cardiovascular surgery [23], [24] and neurosurgery [25] because the empirical models (which are translated into mathematical criteria suitable for optimization) are considered well established. Shamir et al. [26] reported a preoperative planning method for selecting the safest insertion path for straight tools in image-guided keyhole neurosurgery. With their method, the trajectory risk was evaluated based on the uncertainty in the tool placement, the proximity of critical brain structures and on a predefined table of quantitative geometric risk measures. Essert et al. [27] reported an optimal electrode trajectory planning method to assist surgeons in deep brain stimulation. Optimization of possible solutions proceeds using a formal geometric solver using multimodal brain images and a template. To use AP in robot-assisted abdominal interventions, the procedure should be thoroughly studied to establish appropriate empirical models and optimization criteria. The insertion depth, volume of healthy tissue that is ablated and the distances between the needle and important organs are candidates for optimization criteria. Baegert [28], [29] and Villard [30] reported an automatic insertion-trajectory planning method based on hard constraints, which was used to determine the insertion zones on the skin. A given needle-insertion trajectory can then be rated using a weighted sum that combines several soft constrains. However, some researchers have pointed out that the weighted sum rating may be misleading [31]. Moreover, the precision of the proposed algorithms is determined by the discrete size of the surface, and an exact mathematical description of the insertion zones cannot be obtained. Seitel et al. [31] extended the work of Baegert and Villard to achieve a fast and robust implementation for automatic or semi-automatic trajectory planning. The Pareto optimality was used to consider insertion trajectories without requiring weightings. Furthermore, the planning results were based on mesh points of critical structures; therefore, the resolution of the mesh should be carefully chosen to balance the runtime and planning accuracy. Moreover, the method was limited by the resolution of the screen that was used. Schumann et al. [32], [33] developed a method, in which a feasible insertion path for a given target can be obtained based on techniques from the fields of computer graphics and image processing, using the path constraints. Kapoora et al. [34] developed an automatic surgical planning scheme by integrating virtual reality, haptic feedback tools and real-time deformation analysis of soft tissues, which enhances the realism of planning tool. With the help of surgical planning, the optimal needle-insertion path can be obtained. Chen et al. [35] reported a method for searching electrode placements, which couples finiteelement method models of RFA with an optimization strategy. Hasse et al. [36] reported an optimization procedure to determine optimal probe positions with RFA surgery. Using semi-finite constraints, they ensured that the probe did not harm high-risk structures. Jaberzadeh et al. [37] developed an automatic preoperative path planning algorithm to propose a placement for multiple needles in $3 \mathrm{D}$. The location of liver, tumour, skin and vessels is reconstructed firstly. And then the needle tip is placed at an initial position manually. In reference [37], through, optimization process, the needles' placements are determined with the optimization objective which is to maximize the amount of frozen tumour and minimize non-damaged tumour parts and damaged healthy tissue. However, a set of feasible insertion paths was not obtained, and the constraints on the motion of the robot were not considered during the surgical planning. The solution set is useful for adjusting the planned insertion path to satisfy some requirements during the surgery. For example, placement of the US probe should enable monitoring of the process of insertion to ensure safety during surgery [2]. With the help of the solution set, the surgeon can easily adjust the planned insertion path to satisfy these requirements. 


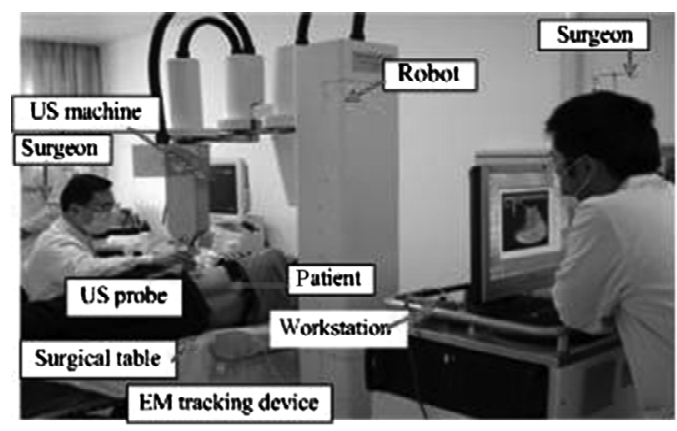

(a)

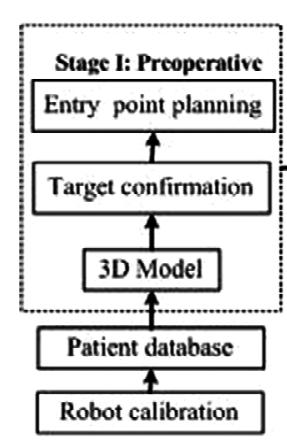

Robot calibration

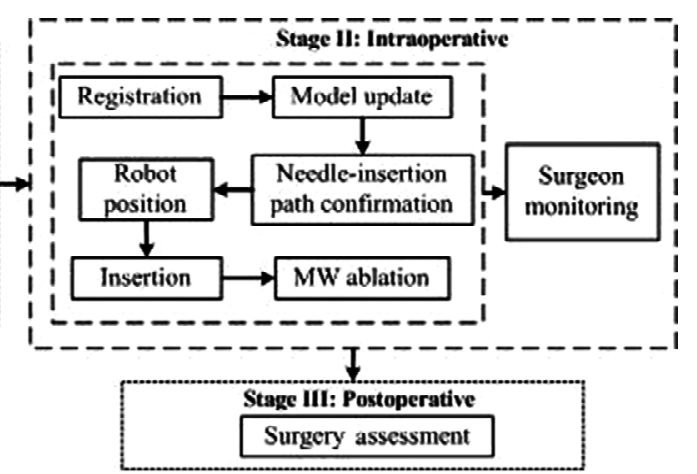

(b)

Figure 2. A flow chart describing the operation of the RALTCT system: (a) surgery environment and (b) operation process.

According to the above analysis, SAP or AP is more suitable for MW ablation of liver tumours surgery as the interaction between multiple manipulators of the robot and the deformation of soft tissue during insertion should be considered. In addition, a set of feasible insertion paths is useful for surgeon to choose an available insertion path or adjust the planned path. Therefore, in this paper, we propose an SAP planning method. It is a mathematic method of obtaining the set of all feasible needle-insertion paths. Each path in this set should satisfy the clinical requirements and the constraints of the motion of the robot, including avoiding interference with critical structures, and the constraint that the insertion depth should less than the length of the needle and so on. With the aid of the solution set, even an inexperienced surgeon can select a feasible needle-insertion path rapidly. Furthermore, with appropriate optimization criteria, the proposed method can be turned into the AP method to choose the optimal needle-insertion path automatically in the solution set. The contributions of our work are a mathematical description of the critical structures in the abdominal cavity and the analytical solution of the needle collision-free reachable workspace (CFRW) used to verify that the needle-insertion path within it will not interfere with the ribs or primary blood vessels and could meet the clinical needs.

\section{Materials and Methods}

\subsection{Operating Procedure and the Constrains for Surgical Planning}

Before discussing our approach to preoperative planning, it is necessary to describe the operating procedure of the RALTCT system to clarify the constraints and the purpose of the preoperative planning. In the following, we describe the operating procedure in detail.

The procedure of the RALTCT system can be divided into three steps: preoperative surgical planning, intraoperative therapy and postoperative assessment [2]. Figure 2 shows a flow chart describing this process.

During the preoperative step, the tasks include the calibration of the robot, a two-dimensional (2D) liver tumour scan, 3D liver tumour model reconstruction, target point selection (the optimal location to destruct the entire malignant liver tumour specified by the surgical navigation software), and planning of the needle-insertion path, which depends on the surgeon's experience and the reconstructed 3D operating environment. It should be noted that the planned needle-insertion path should avoid any vital organs or tissues (e.g., primary blood vessels or ribs).

In the intraoperative step, the first task is to transform the different coordinate systems (e.g., the robot frame, the physical patient frame and the $3 \mathrm{D}$ reconstructed model frame) into a uniform frame (generally the robot frame) using an electro-magnetic (EM) tracking device, such as the Ascension Bird ${ }^{\mathrm{TM}}$ position sensor of the RALTCT system. The planned needle-insertion path is then mapped to the uniform frame such that the robot can move to the desired pose (position and orientation) according to the calculated joint angles corresponding to the planned needle-insertion path. The needle is then inserted manually through a needle-guiding device at the end of the robot, and at the same time, the treatment is conducted with real-time monitoring of the surgical navigation software to ensure safety. It is should be noted that the patient must hold their breath during insertion to maintain the body static, resulting in better operation accuracy.

In the postoperative step, the surgeon collects surgical information to evaluate the effectiveness of the operation.

Clearly planning of the needle-insertion path is important in the RALTCT system for MW ablation of malignant liver tumours. With the RALTCT system, preoperative planning focuses on the needle-insertion path planning. The abdominal operation environment is complicated. As shown in Fig. 3, there is much important tissue and many organs. The basic requirements of the needleinsertion path are that the path should avoid interference with these important anatomical structures, to meet the needle kinematic constraints, and to guide the tip of the needle precisely to the target. Therefore, the constraints for the insertion path planning include the following: (1) The needle-insertion path should avoid interfering with the obstacles in the abdominal operation environment, such as blood vessels, ribs. Because unavoidable deviation from a planned path in practice may cause a fatal injury, needleinsertion paths that are very close to vital organs should be abandoned. (2) To destroy the entire tumour with the minimum damage and decrease the recurrence rate, 


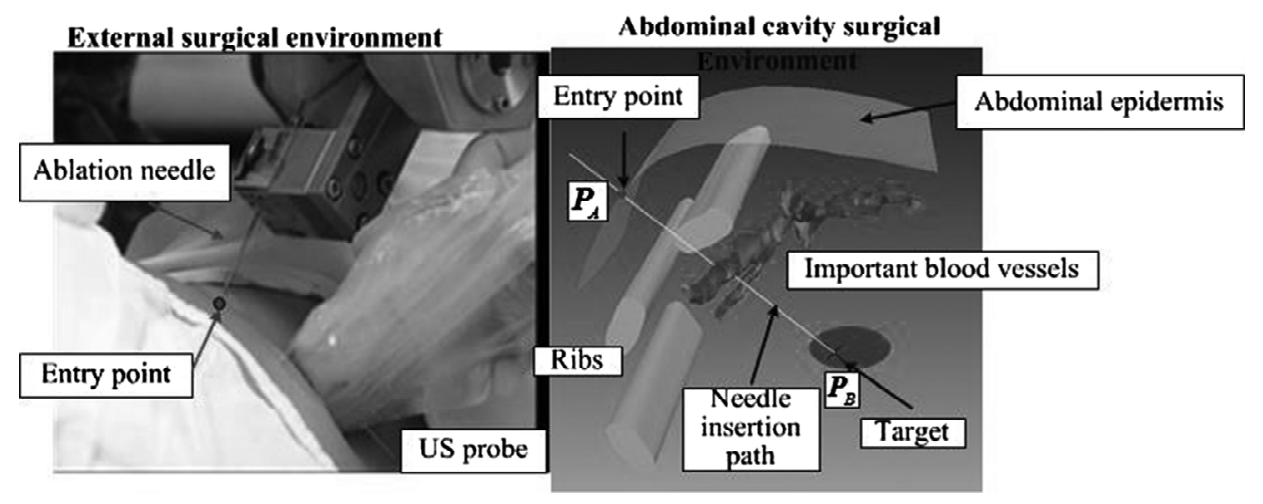

Figure 3. A schematic diagram of the abdominal operation environment.

the tip of the needle should reach the target in the tumour accurately. (3) The insertion path should be shorter than the length of the needle, and (4) the needle-insertion path should meet the movement capabilities of the robot. In other words, the planned insertion path can be carried out by the robot.

Traditionally, planning is performed based on 2D slices of $\mathrm{CT}$ or magnetic resonance imaging scans. However, the identification of an optimal access path to the target under consideration of the abovementioned constraints can be challenging using visualization alone. Note that, currently, the needle-insertion path is determined manually, and hence relies on the surgeon's skill; the accuracy of the operation is therefore dependent on the skill of the surgeon, which limits the applications and increases the surgeon's workload. Additional, the identification of an optimal access path to the target under consideration of the abovementioned criteria can be challenging using the traditional visualization alone. To address this, in this paper we propose an approach to needle-insertion path planning.

In summary, our proposed preoperative planning method is expected to aid the surgeon (especially inexperienced surgeons) in rapidly selecting a feasible needleinsertion path that satisfies the aforementioned basic requirements. To achieve the proposed preoperative planning capabilities, we solve the CFRW of the needle, determine a pose set, which the needle can reach without interference between the needle and the obstacles, and which satisfies the clinical constraints and the constraints of the motion of the robot. The surgeon may then select a needle-insertion path within the CFRW of the needle easily. The advantage is that the needle-insertion path in the CFRW can avoid collisions with structures in the body, and can satisfy the clinical constraints.

\subsection{Physical Model of the Needle}

First, we introduce a simplified physical model of the needle, which is the foundation of our preoperative planning strategy. With the RALTCT system, the desired pose of the robot depends on the entry point $P_{A}$ on the patient's abdominal epidermis and the target point $P_{B}$ in the malignant tumour; therefore, the line connecting points $P_{A}$ and $P_{B}$ is the needle-insertion path. The target point $P_{B}$ is automatically calculated using the surgical navigation software, where the area of the malignant tumour is extracted from the scanned US images [2]. Therefore, the free parameter of the needle-insertion path is the entry point $P_{A}$, and so needle-insertion path planning becomes the problem of determining the entry point $P_{A}$. With such problems, physical models have been used previously, whereby the target is generally regarded as a fixed point in the space. The task then becomes determining the entry point on the skin. For example, Seitel et al. [31] simplified the physical model as a virtual camera fixed on the target. This camera had a $360^{\circ}$ panoramic view, and the patients' epidermis celiac, which can be seen from the camera, was the entry point region corresponding to the feasible needle-insertion path. The potential constraints of the model are that the line-of-sight to the camera is not blocked by the important structures within the abdominal cavity, and that the needle-insertion paths are straight lines. Schumann's research [32] was also based on a given lesion target. A cylindrical projection method was used, whereby the centre point was fixed on the lesion target. The puncture paths were discrete, and the constraints and feasible needle-insertion paths were determined. However, none of these methods can obtain accurate mathematical expressions for the feasible solution set of needle-insertion paths.

In this work, an analytical method is proposed to obtain the precise solution set of the feasible needle-insertion paths. A physical model of the inserted needle is then simplified as follows. Because the target point $P_{B}$ is fixed, the inserted needle can be regarded as an arm with a single ball-hinge joint, that has three degrees of freedom (DOF), and whereby the centre coincides with the target point $P_{B}$ (Fig. 4). Because rotation of the needle about its axis will not affect the needle pose, the needle pose can be represented by the other two rotational DOF. From the above analysis, the target $\boldsymbol{P}_{B}$ is known at a given fixed point in the space, and the entry point $\boldsymbol{P}_{A}$ is the planning object. The movement poses of the arm correspond to the needle-insertion paths. Each pose of the arm corresponds to the entry point $\boldsymbol{P}_{A_{n}}(n=1,2, \ldots,+\infty)$ on the abdominal epidermis. The solution set of the feasible needle-insertion path is a set of poses of the arm that do not interfere with 


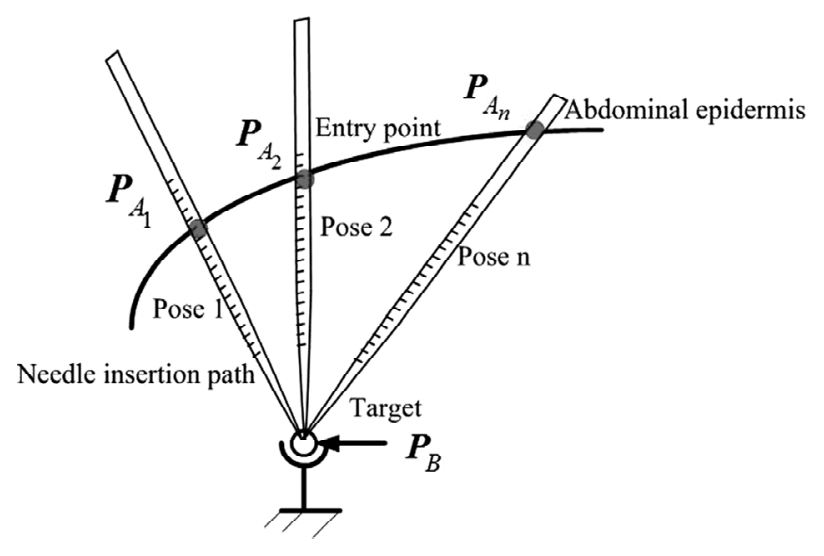

Figure 4. Physical model of the needle.

any obstacles (i.e., blood vessels and ribs) and meet the clinical constrains; this is the CFRW of the arm.

\subsection{Analytical Description of the Boundary of the Reachable Workspace of the Needle with No Obstacles}

Based on the single ball-hinge joint model of the needle described in the previous section, here we provide an analytical description of the boundaries of the reachable workspace of the needle with no obstacles, which is based on our previous work [38]. An analytical description of the needle CFRW will then be analysed in detail in the following section, where the obstacles (i.e., primary blood vessels and ribs) are introduced to the surgical planning environment.

We set up a global coordinate system $\Sigma$, where the $z$-axis is vertical and the origin coincides with $P_{B}$, as shown in Fig. 5. Because rotation of the needle about its own axis does not affect the pose of the needle, the pose can be represented by the rotation angles $\theta$ about the $y$-axis and $\varphi$ about the $z$-axis. In the physical model of the needle, the needle-insertion path may be considered as the pose of the arm that corresponds to the angles $\theta$ and $\varphi$, and the entry point is the intersection between the needle-insertion path and the abdominal epidermis of the patient. Because of the limited space of the abdomen and the length of the inserted needle $r$, the angles $\theta$ and $\varphi$ are both finite in the global coordinate system $\Sigma$. Without considering the obstacles (i.e., primary blood vessels and ribs), the workspace of the needle-insertion path will be a 3D cone, as shown in Fig. 5, where the boundaries of the workspace in each 2D $x y$ plane layered along the $z$-axis are circles. When the obstacles are taken into account, we must solve the needle CFRW in the global coordinate system $\Sigma$, which will be analysed in detail in the following section.

In the coordinate system $\sum$, the generalized coordinates $\boldsymbol{q}=[x, y, z, \theta, \varphi]^{T} \in R^{5}$ are used to describe the position and orientation of the needle, and the end-point of the needle is given by $\boldsymbol{P}_{A}=[x, y, z]^{T} \in R^{3}$. In the system where the needle can move around the ball-hinge joint, the output coordinates' dimensions of the generalized coordinates are $n_{u}=3$. The number of dimensions $\partial A$ of the

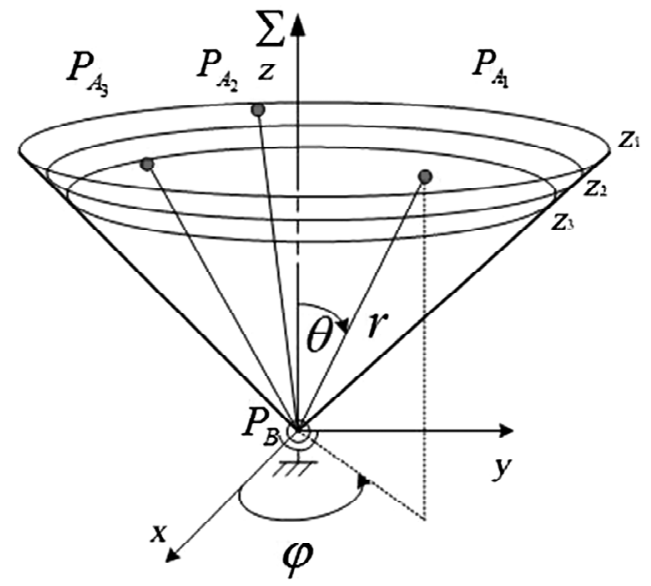

Figure 5. The global coordinate system of the needle $\Sigma$.

boundaries of the reachable workspace of the needle is $n_{u}-1=2$. Therefore, $\partial A$ is a $2 \mathrm{D}$ surface in threedimensional (3D) space. To simplify the calculations, the boundaries are decomposed into a group of layers along the $z$-axis in the global coordinate system $\Sigma$. In each $x y$ planar layer, the boundary is a 1D manifold. In this case, the boundary in the $3 \mathrm{D}$ space is considered a group of $1 \mathrm{D}$ manifolds. As shown in Fig. 5, for a given $x y$ plane, the needle may be considered a telescopic link. For the different poses of the needle, the inserted length $r$ is a variable and only relates to the rotation angles $\theta$. This is consistent with the actual physical insertion properties of the needle during surgery; i.e., the length of the needle into the skin differs when the insertion angle varies.

To simplify the calculations, the boundaries of the reachable workspace of the needle are decomposed into a group of layers to be solved. The $x y$ plane with $z_{1}=h, h \in R^{+}$is described as the $z_{1}$ plane, as shown in Fig. 5, and then the coordinates $x, y$ of the intersection point $P_{A}$ between the needle and the $z_{1}$ plane can be represented as a function of the angles of rotation $\theta$ and $\varphi$ at the needle pose $q$ as follows:

$$
\left\{\begin{array}{l}
x=h \times \tan \theta \times \cos \varphi \\
y=h \times \tan \theta \times \sin \varphi
\end{array}\right.
$$

The following kinematic constraints in the global coordinate system $\Sigma$ are applied:

$$
\Phi=\left[\begin{array}{l}
x-h \times \tan \theta \times \cos \varphi \\
y-h \times \tan \theta \times \sin \varphi
\end{array}\right]=0
$$

where $x, y, \theta$ and $\varphi$ are variables and $h$ is a constant.

The parameters $\theta$ and $\varphi$ are subject to the following constraints:

$$
\begin{aligned}
& \theta_{\min } \leq \theta_{j} \leq \theta_{\max } \\
& \varphi_{\min } \leq \varphi_{j} \leq \varphi_{\max }
\end{aligned}
$$


The limits of the two parameters are set based on the clinical constraints and constraints of the motion of the robot. For example, there must always be a safety margin around target, and the length of inserted needle should be shorter than the needle length. Based on the US image of the patient and the 3D reconstructed model of liver, the constraints can be translated into the limits of these two parameters. Based on the structure of the robot, we know that the insertion angle, which is determined by the two parameters $\theta$ and $\varphi$, is also limited by joints four and five of the robot [2]. The limits of these two parameters can be calculated from the robot kinematics, and the final parameters $\theta_{\min }, \theta_{\max }, \varphi_{\min }$ and $\varphi_{\max }$ can be determined by integrating all of the constraints.

To transform the inequality constraints to equality constraints, the unconstrained variables $v_{1}$ and $v_{2}$ are introduced; (3) is then rewritten as follows:

$$
\begin{aligned}
\theta_{j} & =\frac{1}{2}\left(\theta_{\max }+\theta_{\min }\right)+\frac{1}{2}\left(\theta_{\max }-\theta_{\min }\right) \sin w_{1} \\
\varphi_{j} & =\frac{1}{2}\left(\varphi_{\max }+\varphi_{\min }\right)+\frac{1}{2}\left(\varphi_{\max }-\varphi_{\min }\right) \sin w_{1}
\end{aligned}
$$

By substituting this expression into (2), we may eliminate the inequalities through the use of the variables $v_{1}$ and $v_{2}$.

In the $z_{1}$ plane, the output coordinates are $\boldsymbol{u}=[x, y]^{T}$, and $\boldsymbol{v}=\left[v_{1}, v_{2}\right]^{T}$ are the input coordinates. The intermediate coordinates are $\boldsymbol{w}=\varnothing$ and $\boldsymbol{z}=\left[\boldsymbol{w}^{T}, \boldsymbol{v}^{T}\right]^{T}=\boldsymbol{v}$. Based on the kinematic constraint equation $\boldsymbol{\Phi}$ and the vector $\boldsymbol{z}$, the sub-Jacobian matrix $\boldsymbol{\Phi}_{z}$ (i.e., the Jacobian of $\boldsymbol{\Phi}$ with respect to $\boldsymbol{z}$ ) can be solved. We then arrive at an analytical description of the boundaries of the reachable workspace of the needle with no obstacles; i.e.,

$$
\mathbf{G}(\boldsymbol{x}) \equiv\left[\begin{array}{c}
\boldsymbol{\Phi}(\boldsymbol{u}, \boldsymbol{z}) \\
\boldsymbol{\Phi}_{z}^{T}(\boldsymbol{u}, \boldsymbol{z}) \boldsymbol{\zeta} \\
\boldsymbol{\zeta}^{T} \boldsymbol{\zeta}-1
\end{array}\right]=0
$$

where $\boldsymbol{\zeta} \in \mathbb{R}^{2}, \boldsymbol{x}=\left[\boldsymbol{u}^{T}, \boldsymbol{z}^{T}, \boldsymbol{\zeta}^{T}\right]^{T}$. This equation contains five expressions and six variables, and the number of dimensions of $\partial A$ is one.

An analytical description of the boundaries of the reachable workspace of the needle in the other $x y$-planar layers can be obtained using the same method, only by changing the value of $h$. Therefore, the set of $1 \mathrm{D}$ boundaries at each $x y$ plane is the $3 \mathrm{D}$ boundary of the reachable workspace of the needle.

\subsection{Analytical Description of the Boundary of the Needle CFRW}

The environment of the abdominal operation is complicated. There are many important tissues and organs around the liver, which are located in a narrow space, some of which are located under the ribs. In the liver, there resides the portal vein and other primary blood vessels. The needle-insertion path must avoid interference with the

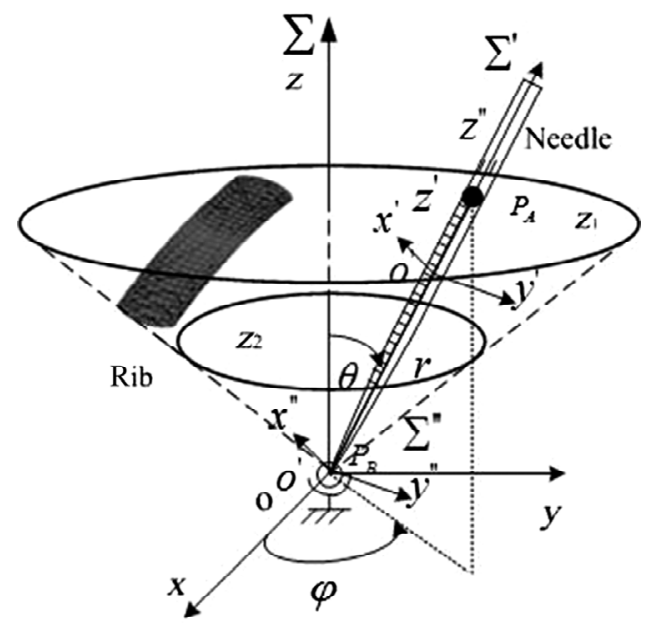

Figure 6 . The global coordinate system $\Sigma$ and the local coordinate system $\Sigma^{\prime}$.

primary blood vessels and ribs to ensure safety of the operation. In our simplified mathematical model, primary blood vessels and ribs are considered as obstacles, and the needle is considered as an arm with two DOFs and a single ball-hinge joint (note that we have neglected the DOF due to rotation about its own axis because of the symmetry of the needle). Using the mathematical model, we can describe the complex physical environment. Because of the variations among patients (such as the location and shape of primary blood vessels and ribs), here we describe a generalized method to obtain an analytical description of the boundaries of the needle CFRW. The criterion is the basis for the computation and visualization of the boundary surfaces, while the boundary surface is the basis of the optimal needle-insertion path in the preoperative planning. The primary benefit of this approach is that the analytical criterion of the boundaries is convenient for the subsequent selection of the needle-insertion path.

The mathematical descriptions of the needle and the obstacles (ribs and primary blood vessels) are the prerequisites of the boundary of the needle CFRW. The needle is described as a cylinder, whereas a third-order continuously differentiable super-quadric [39], [40] is employed to represent the obstacles.

The mathematical description of the needle segment inserted within the abdominal cavity is represented. The mathematical description of the needle varies with different poses, and so we use a local coordinate system $\Sigma^{\prime}$ for the needle, where the $z^{\prime}$-axis coincides with the axis of the needle and the origin $o^{\prime}$ corresponds to the centre of the needle, as shown in Fig. 6.

The curved surface of the needle can be represented by a super-quadric, with a canonical form as in (6) in the local coordinate system $\Sigma^{\prime}$, which is attached to the geometric centre of the needle, i.e.,

$$
\begin{aligned}
f_{\text {needle }}^{\prime}\left(\left[b_{x}^{\prime}, b_{y}^{\prime}, b_{z}^{\prime}\right]^{T}\right)= & \left(b_{x}^{\prime} / 60\right)^{8}+\left(b_{y}^{\prime} / 0.1\right)^{2} \\
& +\left(b_{z}^{\prime} / 0.1\right)^{2}-1,000=0
\end{aligned}
$$

where $\left[b_{x}^{\prime}, b_{y}^{\prime}, b_{z}^{\prime}\right]^{T}$ is the point coordinate for the needle curved surface in $\Sigma^{\prime}$. 
We consider the local coordinate system of the needle $\Sigma^{\prime \prime}$, as shown in Fig. 6, which varies with the motion of needle. The $z^{\prime \prime}$-axis of the coordinate system $\Sigma^{\prime \prime}$ coincides with the axis of needle, and the origin $o^{\prime \prime}$ coincides with the tip of the needle and also with the target point $P_{B}$. Let $T$ be the homogeneous transformation matrix between the local coordinate system $\Sigma^{\prime}$ and the local coordinate system $\Sigma^{\prime \prime}$; the relationship between the point coordinates $\left[b_{x}^{\prime \prime}, b_{y}^{\prime \prime}, b_{z}^{\prime \prime}\right]^{T}$ in the local coordinate system $\Sigma^{\prime \prime}$ and point coordinates $\left[b_{x}^{\prime}, b_{y}^{\prime}, b_{z}^{\prime}\right]^{T}$ in the local coordinate system $\Sigma^{\prime}$ can be expressed as follows:

$$
\left[b_{x}^{\prime \prime}, b_{y}^{\prime \prime}, b_{z}^{\prime \prime}\right]^{T}=T\left[b_{x}^{\prime}, b_{y}^{\prime}, b_{z}^{\prime}\right]^{T}
$$

Because the length $r$ of the needle is constant, $\boldsymbol{T}$ can be expressed as follows:

$$
\boldsymbol{T}=\left[\begin{array}{llll}
1 & 0 & 0 & 0 \\
0 & 1 & 0 & 0 \\
0 & 0 & 1 & r / 2 \\
0 & 0 & 0 & 1
\end{array}\right]\left[\begin{array}{cccc}
\cos (\pi / 2) & 0 & \sin (\pi / 2) & 0 \\
0 & 1 & 0 & 0 \\
-\sin (\pi / 2) & 0 & \cos (\pi / 2) & 0 \\
0 & 0 & 0 & 1
\end{array}\right]
$$

Let $\boldsymbol{T}_{w}$ be the homogeneous transformation matrix from the local coordinate system $\Sigma^{\prime \prime}$ to the global coordinate system $\Sigma$; the point coordinates $\left[b_{x}^{\prime \prime}, b_{y}^{\prime \prime}, b_{z}^{\prime \prime}\right]^{T}$ in the local coordinate system $\Sigma^{\prime \prime}$ can be converted by $\left[b_{x}, b_{y}, b_{z}\right]^{T}$ in the global coordinate system $\Sigma$ as follows:

$$
\left[b_{x}, b_{y}, b_{z}, 1\right]^{T}=T_{w}\left[b_{x}^{\prime \prime}, b_{y}^{\prime \prime}, b_{z}^{\prime \prime}, 1\right]^{T}
$$

The pose of the needle $q$ corresponds to the rotation angles $\theta$ and $\varphi$ in the global coordinate system $\Sigma$; thus, we may obtain the transformation matrix $\boldsymbol{T}_{w}$ as follows:

$\boldsymbol{T}_{w}=\left[\begin{array}{cccc}\cos \varphi & -\sin \varphi & 0 & 0 \\ \sin \varphi & \cos \varphi & 0 & 0 \\ 0 & 0 & 1 & 0 \\ 0 & 0 & 0 & 1\end{array}\right] \times\left[\begin{array}{cccc}\cos \theta & 0 & \sin \theta & 0 \\ 0 & 1 & 0 & 0 \\ -\sin \theta & 0 & \cos \theta & 0 \\ 0 & 0 & 0 & 1\end{array}\right]$

According to (6)-(10), the function $f_{\text {needle }}$ describing the curved surface of the needle in the global coordinate system $\Sigma$ can be obtained.

The points of obstacles (ribs and primary blood vessels) can be obtained from segmentation of the reconstructed 3D model [2]. These sample points are then used to obtain implicit polynomials describing the obstacles, which will be discussed in detail in the following section. To obtain the analytical description of the boundary of the needle CFRW, we take a rib as an obstacle. In this section, the rib is represented by a super-quadric with canonical form [41]; i.e.,

$f\left(\left[a_{x}^{\prime}, a_{y}^{\prime}, a_{z}^{\prime}\right]^{T}\right)=\left(\frac{a_{x}^{\prime}}{a_{1}}\right)^{12}+\left(\frac{a_{y}^{\prime}}{a_{2}}\right)^{2}+\left(\frac{a_{z}^{\prime}}{a_{3}}\right)^{2}-1=0$ where $\left[a_{x}^{\prime}, a_{y}^{\prime}, a_{z}^{\prime}\right]^{T} \in R^{3}$ are the coordinates of a point of the curved surface of the rib in the canonical coordinate system; $a_{1}, a_{2}$ and $a_{3}$ indicate the semi-axis length of the super-quadric along the $x, y$ and $z$ axes, respectively, which can be determined from least squares fitting. Note that $f\left(\left[a_{x}^{\prime}, a_{y}^{\prime}, a_{z}^{\prime}\right]^{T}\right)$ is a third-order continuously differentiable convex function [39].

The location of the rib relative to the target in the global coordinate system $\Sigma$ can be represented using the homogeneous transformation based on the image segmentation results. For this reason, the function $f_{\text {rib }}$ describing the surface of the rib in the global coordinate system $\Sigma$ may be obtained.

Similarly, the other obstacles (primary blood vessels) may also be represented by $f_{o b s}$ as third-order continuously differentiable implicit polynomials.

Once the mathematical descriptions of the obstacles and the needle are obtained, the collision-free equation describing the needle and obstacles can be formulated as follows [38]:

$$
\boldsymbol{\Phi}^{\prime} \equiv\left[\begin{array}{c}
\frac{\partial f_{0}(\eta)}{\partial \eta}+\lambda_{1} \frac{\partial f_{\text {needle }}(q, \eta)}{\partial \eta}+\lambda_{2} \frac{\partial f_{\text {obs }}(\eta)}{\partial \eta} \\
f_{\text {needle }}(q, \eta)+s_{1} \\
f_{\text {obs }}(\eta)+s_{2} \\
L S e-\mu e \\
\left\|a_{g}-b_{g}\right\|_{2}^{2}-\gamma^{2}-s_{d}^{2}
\end{array}\right]=0
$$

where $a_{g}=\left[a_{x}, a_{y}, a_{z}\right]^{T}, \quad b_{g}=\left[b_{x}, b_{y}, b_{z}\right]^{T}, \quad \eta=\left[a_{g}^{T}, b_{g}^{T}\right]^{T}$, $f_{0}(\eta)=\left\|a_{g}-b_{g}\right\|_{2}^{2}$ and $\lambda=\left[\lambda_{1}, \lambda_{2}\right]^{T}$ is a $2 \times 1$ vector of Lagrange multipliers; $\boldsymbol{L}$ is a $2 \times 2$ diagonal matrix of $\lambda_{1}$ and $\lambda_{2} ; \boldsymbol{S}$ is a $2 \times 2$ diagonal matrix containing the slack variables $s_{1}$ and $s_{2} ; e=[1,1]^{T} ;$ and $\gamma>0$ is the safedistance parameter. It is important to minimize the risk of fatal injuries, and hence, $\gamma$ can be determined according to clinical requirements. The term $\mu$ is the barrier parameter and $s_{d}$ is a slack variable. Note that (12) is composed of 11 expressions with 11 variables, and $w_{1}=\left[\eta^{T}, \lambda^{T}, s^{T}, s_{d}\right]^{T}$.

It appears that the boundaries of the CFRW of the needle can also be described as a 3D surface. To simplify the calculations, these boundaries are also decomposed into a group of layers along the $z$-axis in the global coordinate system $\Sigma$. In each $x y$ planar layer, the boundary is a 1D manifold, and the boundaries in the $3 \mathrm{D}$ space can be considered as a group of $1 \mathrm{D}$ manifolds. The equations describing the kinematic constraints of the needle in the global coordinate system $\Sigma$ in the $z_{1}$ plane were established in the previous section.

By combing (2) and (12), we may obtain the following extended set of constraint equations:

$$
\tilde{\Phi} \equiv\left[\begin{array}{c}
\Phi \\
\Phi^{\prime}
\end{array}\right]=0
$$


The analytical criterion describing of the boundaries of the needle CFRW can be expressed as follows:

$$
\tilde{\mathbf{G}}(\tilde{x}) \equiv\left[\begin{array}{c}
\tilde{\Phi}(u, \tilde{z}) \\
\tilde{\Phi}_{\tilde{z}}^{T}(u, \tilde{z}) \tilde{\xi} \\
\tilde{\xi}^{T} \tilde{\xi}-1
\end{array}\right]=0
$$

where $\quad u=[x, y]^{T}, \quad \tilde{z}=\left[a_{x}, a_{y}, a_{z}, b_{x}, b_{y}, b_{z}, \lambda_{1}, \lambda_{2}, s_{1}, s_{2}\right.$, $\left.s_{d}, v_{1}, v_{2}\right]^{T}, \tilde{x}=\left[\mathrm{u}^{T}, \tilde{z}^{T}, \tilde{\xi}^{T}\right]^{T} \in R^{28}$ and $\tilde{\xi}$ is a unit vector with dimensionality 13. As (14) contains 27 expressions, $\tilde{\mathbf{G}}(\tilde{x}): R^{28} \rightarrow R^{27}$.

The boundaries of the needle CFRW are obtained as a $1 \mathrm{D}$ manifold in the $z_{1}$ plane. The boundaries of the needle CFRW in the other $z$ planes may be obtained in the same way, except for the adjusted parameter $h\left(h \in\left[0, h_{\max }\right]\right.$, where $h_{\max }$ is determined based on the clinical conditions). The 3D boundary of the needle CFRW comprises a group of boundaries of the needle CFRW in the $z$ planes. A line that connects any of the points on the boundaries on any of the $z$ planes with $P_{B}$ will not interfere with obstacles and also meet the clinical constrains.

\subsection{Mathematical Description of the Obstacles}

In this section, we use implicit polynomials to describe the obstacles (i.e., blood vessels and ribs) in the surgical environment of the abdominal cavity, as well as the abdominal epidermis. The mathematical descriptions of those objects provide constraints for surgical planning of the medical robotic.

As the sample points of obstacles are obtained from segmentation of the reconstructed 3D model [2], the fitting problem is as follows: given a set of points, find an analytic surface that passes close to these points. Based on the definition of the implicit polynomials, the surface of the objects in the space can be described as zero-sets of the smooth function $f: R^{3} \rightarrow R^{1}$; i.e., $Z(f)=\{x: f(x)=0$, $\left.x \in R^{3}\right\}$, which has the following properties:

$$
\left\{\begin{array}{l}
\left\{x \mid f(x)=0, x \in \mathbb{R}^{3}\right\}: \text { denotes the objects' surface } \\
\left\{x \mid f(x)>0, x \in \mathbb{R}^{3}\right\}: \text { denotes the outside of objects } \\
\left\{x \mid f(x)<0, x \in \mathbb{R}^{3}\right\}: \text { denotes the inside of objects }
\end{array}\right.
$$

To obtain the implicit polynomials $Z(f)$, we let $y=f(x)$ be a single-variable function, where $x$ can be any point, $f(x) \neq 0$, and $z$ is the closest point on $f$ to $x$; we then obtain

$$
0=f(z) \approx f(x)+f^{\prime}(x)(z-x)
$$

therefore, we may write $(x-z)^{2} \approx[f(x)]^{2} /\left[f^{\prime}(x)\right]^{2}$, where $(x-z)^{2}$ is the squared distance from $x$ to the zero-set of $f$. This conclusion can be generalized to describe the squared distance for any point to the 3D surface [42]. Assuming that the implicit polynomials describing the surface of the obstacles is given by $C=\{x: f(x)=0\}$, we may write

$\operatorname{distance}(x, C)^{2} \approx \boldsymbol{F}(x)^{T}\left[\left(\frac{\partial \boldsymbol{F}(x)}{\partial x}\right)\left(\frac{\partial \boldsymbol{F}(x)}{\partial x}\right)^{T}\right]^{-1} \boldsymbol{F}(x)$

where $\boldsymbol{F}(x)=(\boldsymbol{f}(x))^{T}$ and $\boldsymbol{F}(x) / \partial x$ is a $1 \times 3$ Jacobian matrix. Equation (17) may also be applied to the situation whereby $f(x): R^{n} \rightarrow R^{k}$, where $k \leq n$ and where the rank of the $k \times n$ matrix $\partial \boldsymbol{F}(x) / \partial x$ is $k$.

The implicit polynomial $f(x)$ may be written as follows:

$$
f(x)=\boldsymbol{F} \boldsymbol{X}
$$

where $\boldsymbol{F}$ is a coefficient matrix and $\boldsymbol{X}$ is the corresponding polynomial vector. For example, when we choose a quadratic polynomial to describe the surface of the obstacles in the surgical environment, the implicit polynomials may written as

$$
\left\{\begin{array}{c}
\boldsymbol{X}=\left[\begin{array}{lllllllllll}
1 & z & y & x & z^{2} & z y & z x & y^{2} & y x & x^{2}
\end{array}\right]^{T} \\
\boldsymbol{F}=\left[\begin{array}{llllllllll}
a_{1} & a_{2} & a_{3} & a_{4} & a_{5} & a_{6} & a_{7} & a_{8} & a_{9} & a_{10}
\end{array}\right]
\end{array}\right.
$$

By substituting (18) into (19), assuming that the point set on the surface of the obstacles is given by $P=\left\{x_{1}, \ldots, x_{p}\right\}$ we obtain

$$
\operatorname{distance}(x, C)^{2} \approx \boldsymbol{X}^{T} \boldsymbol{F}\left[\boldsymbol{F}\left(\frac{\partial \boldsymbol{X}}{\partial x}\right)\left(\frac{\partial \boldsymbol{X}}{\partial x}\right)^{T} \boldsymbol{F}^{T}\right]^{-1} \boldsymbol{F} \boldsymbol{X}
$$

From (17), the approximate mean of the squared distance from $P$ to $f(x)$ is given by

$$
\Phi_{p}(\boldsymbol{F})=\frac{1}{p} \sum_{1 \leq i \leq p}\left\{\boldsymbol{X}^{T} \boldsymbol{F}\left[\boldsymbol{F}\left(\frac{\partial \boldsymbol{X}}{\partial x}\right) \quad\left(\frac{\partial \boldsymbol{X}}{\partial x}\right)^{T} \boldsymbol{F}^{T}\right]^{-1} \boldsymbol{F} \boldsymbol{X}\right\}
$$

however, the computational burden required to minimize $\Phi_{p}(\boldsymbol{F})$ is considerable. By substituting a constant ma$\operatorname{trix}(1 / p) \sum_{1 \leq i \leq p}\left\{(\partial \boldsymbol{X} / \partial x)\left(\partial \boldsymbol{X}^{T} / \partial x\right)\right\}$ for variable matrix $(\partial \boldsymbol{X} / \partial x)(\partial \boldsymbol{X} / \partial x)^{T}$, the approximate mean squared distance can be obtained as follows:

$$
\begin{aligned}
& \xi_{p}(\boldsymbol{F}) \\
& =\frac{1}{p} \sum_{1 \leq i \leq p}\left\{\boldsymbol{X}^{T} \boldsymbol{F}\left[\boldsymbol{F}\left(\frac{1}{p} \sum_{1 \leq i \leq p}\left\{\frac{\partial \boldsymbol{X}}{\partial x} \frac{\partial \boldsymbol{X}^{T}}{\partial x}\right\}\right) \boldsymbol{F}^{T}\right]^{-1} \boldsymbol{F} \boldsymbol{X}\right\}
\end{aligned}
$$


i.e., $\xi_{p}(\boldsymbol{F})$ is the approximate mean squared distance from $P$ to the surface $f(x)$ along the mean normal direction to the surface. When the set of points describing the surface of the obstacles is given, and the order of the polynomial is known, the fitting to the surface of the obstacles can be converted to minimize $\xi_{p}(\boldsymbol{F})$. The order of the implicit polynomials describing the surface of the obstacles in the surgical environment can then be obtained using the following method.

The order of the fitting polynomials should be as low as possible, because this reduces the computational complexity and also avoids extraneous components that may deviate from the surface of the obstacles [43].
Bezout's theorem can be used to determine the order of the implicit polynomials. Let $C_{n}$ be the zero-sets of the $n$-th-order polynomial, and let $C_{m}$ be the zero-sets of the $m$-th-order polynomial. If the two polynomials have no common component, the two zero-sets cannot meet at more than $n \times m$ points.

Because the ribs and blood vessels are curved structures, and the blood vessels may bifurcate, the number of intersection points between an obstacle and a line is four. From Bezout's theorem, as the order of linear polynomial is one, the order of the implicit polynomials should be four. The form of the implicit polynomials describing the obstacles can be written as follows:

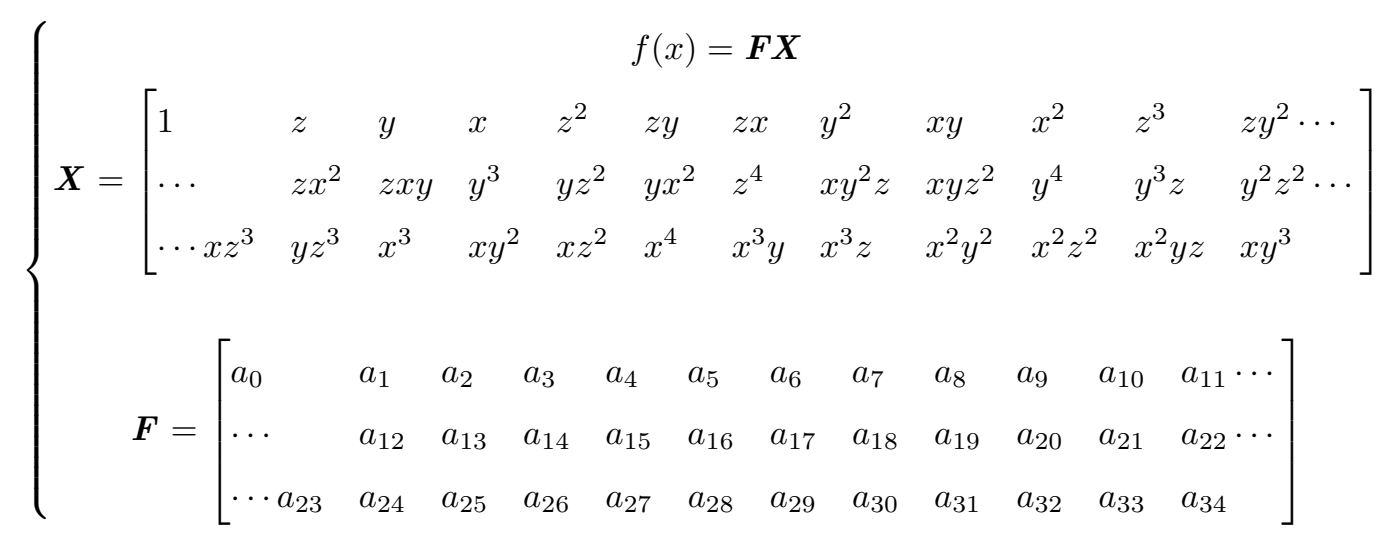

Given the set of points and the degree of the polynomials, the process of surface estimation becomes fitting to the parameter matrix $\boldsymbol{F}$ to minimize $\xi_{p}(\boldsymbol{F})$. A nonlinear least-squares algorithm [44] can be used to solve this problem; however, there are a number of shortcomings with this approach. For example, it is sensitive to the initial value, and a poor initial value may cause the result to fail to converge. For these reasons, we chose another fast method to solve this problem, which is not sensitive to the initial value and better higher precision.

Based on the implicit polynomial describing the ribs and blood vessels (equation (23)), we denote $\boldsymbol{M}=(1 / p)$ $\sum_{1 \leq i \leq p} \boldsymbol{X} \boldsymbol{X}^{T}$ and $\boldsymbol{N}=(1 / p) \sum_{1 \leq i \leq p}\left\{(\partial \boldsymbol{X} / \partial x)\left(\partial \boldsymbol{X}^{T} / \partial x\right)\right\}$, where $\boldsymbol{N}$ is the identity matrix. It has been shown [45] that the parameter matrix $\boldsymbol{F}$ that minimizes $\xi_{p}(\boldsymbol{F})$ is the eigenvectors of $\boldsymbol{M}$ corresponding to the smallest eigenvalue.

The algorithm to obtain the parameter matrix $F$ describing the implicit polynomials of the obstacles is as follows:

1. Compute the diagonal decomposition of $\boldsymbol{N}=\boldsymbol{U}^{T} \boldsymbol{D} \boldsymbol{U}$, with $\boldsymbol{U} \boldsymbol{U}^{T}=\boldsymbol{I}, \boldsymbol{D}=\operatorname{diag}\left(d_{1}, \ldots, d_{m}\right)$ and $d_{1} \geq \cdots \geq$ $d_{s}>d_{s+1}=\cdots=d_{m}=0$.

2. Let $\boldsymbol{U}_{1}, \cdots, \boldsymbol{U}_{m}$ denote the rows of $\boldsymbol{U}$, and let

$$
\boldsymbol{H}=\left[\begin{array}{c}
\boldsymbol{U}_{s+1} \\
\vdots \\
\boldsymbol{U}_{m}
\end{array}\right], \quad \boldsymbol{G}=\left[\begin{array}{c}
\frac{1}{\sqrt{d_{1}}} \\
\vdots \\
\frac{1}{\sqrt{d_{s}}}
\end{array}\right]\left[\begin{array}{c}
\boldsymbol{U}_{1} \\
\vdots \\
\boldsymbol{U}_{s}
\end{array}\right] .
$$

3. Compute $\boldsymbol{T}=\boldsymbol{M} \boldsymbol{H}^{\boldsymbol{T}}\left[\boldsymbol{H} \boldsymbol{M} \boldsymbol{H}^{T}\right]^{-1} \boldsymbol{H}$.

4. Compute $\boldsymbol{L}=\boldsymbol{G}[\boldsymbol{I}-\boldsymbol{T}] \boldsymbol{M} \boldsymbol{G}^{T}$.

5. Compute the eigenvectors of $\boldsymbol{L}$, where $\boldsymbol{\lambda}_{1}^{T}$ corresponds to the smallest eigenvalue.

6. Compute $\boldsymbol{F}=\boldsymbol{\lambda} \boldsymbol{G}[\boldsymbol{I}-\boldsymbol{T}]$.

The implicit polynomials describing the obstacles can be obtained based on the sample data set. These results can be used to provide constraints to solve the boundaries of the needle CFRW.

\subsection{Closest Distance between the Needle-Insertion Path and the Critical Structures}

Once the CFRW of the needle has been obtained, the surgeon may select a feasible needle-insertion path. The clinical constraints and the constraints on the motion of the robot, including the insertion angle and insertion length has been considered to solve the needle CFRW. During surgery, needle-insertion paths that pass close to the critical structures are considered dangerous. Therefore, we develop an intraoperative risk analysis to obtain the closest distance from the needle-insertion path to the critical structures to rate the selected path. The core of surgical risk analysis is calculating the closest distance between the needle and critical structures, as well as the time parameters when the distance between the needle and the obstacles is shortest. During these critical times, the surgeon should be especially vigilant. In this section, we calculate the times when the distance between the needle and the obstacles is the 
shortest using a non-discrete method. During the insertion of the needle, the critical structures in the abdominal cavity are stationary (relative to the macroscopic motion, soft tissue deformation of the liver caused by the insertion can be neglected). The needle has slow and uniform motion along the needle-insertion path, aided by the guide sleeve at the end of the robot and controlled manually by the surgeon. We assume that the surfaces of the obstacles and the needle can be described by $f_{\mathrm{Obs}}\left(\boldsymbol{O} \boldsymbol{b} s_{l}\right)=0$ and $f_{\text {Needle }}\left(\right.$ Needle $\left._{l}\right)=0$ in their respective local coordinate systems.

Suppose that the needle has uniform linear motion along the direction of the unit vector $\boldsymbol{g}_{\text {Needle }}$, and that the speed is $\boldsymbol{v}_{\text {Needle. }}$. In the time interval [0, $\left.T_{\max }\right]$, the problem of solving the shortest distance between the needle and the obstacles can be converted into a convex optimization problem [39] as follows:

$$
\begin{array}{ll}
\text { Min } & \left\|\boldsymbol{O b s}_{\mathrm{g}}-\boldsymbol{N e e d l e}_{\mathrm{g}}\right\|_{2}^{2} \\
\text { Subject to: } & \boldsymbol{O b s}_{\mathrm{g}}=\boldsymbol{R}_{\text {Obs }} \boldsymbol{O b}_{l}+\boldsymbol{p}_{\text {Obs }} \\
& \text { Needle }_{\mathrm{g}}=\boldsymbol{R}_{\text {Needle }} \text { Needle }_{l}+\boldsymbol{p}_{\text {Needle }} \\
& +\boldsymbol{v}_{\text {Needle }} \boldsymbol{t g}_{\text {Needle }} \\
& f_{\text {Obs }}\left(\boldsymbol{O b s}_{l}\right) \leq 0, f_{\text {Needle }}\left(\text { Needle }_{l}\right) \leq 0 \\
& 0 \leq t \leq \boldsymbol{T}_{\max }
\end{array}
$$

where $\boldsymbol{O} \boldsymbol{b} \boldsymbol{s}_{\mathrm{g}}$ is a point on the obstacle and $\boldsymbol{N e e d l e}_{\mathrm{g}}$ is a point on the needle in the global coordinate system; $\left(\boldsymbol{R}_{\text {Obs }}, \boldsymbol{p}_{\text {Obs }}\right)$ and $\left(\boldsymbol{R}_{\text {Needle }}, \boldsymbol{p}_{\text {Needle }}\right)$ are, respectively, the transformation matrices from the local coordinate system of the obstacles and the needle to the global coordinate system.

We introduce the unconstrained variable $t^{\prime}$ to describe $t$ as an equality constraint, so that $t$ can be expressed as follows:

$$
t=\frac{1}{2} T_{\max }+\frac{1}{2} T_{\max } \sin \left(t^{\prime}\right)
$$

By substituting (25) into (24), and substituting $\boldsymbol{O b s}_{\mathrm{g}}$ and Needle $_{\mathrm{g}}$ into the expressions for $f_{\mathrm{Obs}}\left(\mathrm{Ob} s_{l}\right)$ and $f_{\text {Needle }}\left(\right.$ Needle $\left._{l}\right),(24)$ can be rewritten as follows:

$$
\begin{array}{ll}
\text { Min } & \left\|\boldsymbol{O b}_{\mathrm{g}}-\boldsymbol{N e}_{\text {edle }}\right\|_{2}^{2} \\
\text { Subject to: } & f_{\text {Obs }}\left(\boldsymbol{O b}_{\mathrm{g}}\right) \leq 0, f_{\text {Needle }}\left(\boldsymbol{N}_{\text {eedle }}\right) \leq 0
\end{array}
$$

Then, (26) can be transformed to the form of nonlinear optimization problem as follows:

$$
\begin{array}{ll}
\text { Min } & f_{0}(\boldsymbol{X}) \\
\text { Subject to: } & \boldsymbol{f}(\boldsymbol{X})+\boldsymbol{s}=0, \boldsymbol{s} \geq 0
\end{array}
$$

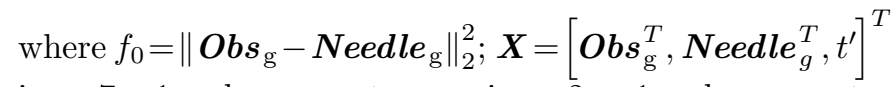
is a $7 \times 1$ column vector; $s$ is a $2 \times 1$ column vector containing the slack variables $s_{1}$ and $s_{2} ; f: R^{6} \rightarrow R^{2}$ is the two inequality constraints in (26).

The Karush-Kuhn-Tucker (KKT) condition [39] describes the necessary and sufficient conditions to solve the shortest distance problem, and the closest points (corresponding to the shortest distance) lie on a curved surface of the two objects. Using the KKT condition, (27) can be expressed as the following set of nonlinear equations:

$$
\begin{aligned}
& \nabla f_{0}(\boldsymbol{X})+(\nabla \boldsymbol{f}(\boldsymbol{X}))^{T} \boldsymbol{\lambda}=0 \\
& \boldsymbol{f}(\boldsymbol{X})+\boldsymbol{s}=0 \\
& \boldsymbol{L S} \boldsymbol{e}=0
\end{aligned}
$$

where $\boldsymbol{\lambda}=\left[\lambda_{1}, \lambda_{2}\right]^{T}$ are the Lagrange multipliers; $L$ is a $2 \times 2$ diagonal matrix containing $\lambda_{1}$ and $\lambda_{2}, S$ is a $2 \times 2$ diagonal matrix of slack variables $s_{1}$ and $s_{2}$, and $e=[1,1]^{T}$.

To avoid the problem whereby the general iterative method depends on the initial value, we choose the interior point method [39], [46] to transfer (28) to the following form:

$$
\begin{aligned}
& \nabla f_{0}(\boldsymbol{X})+(\nabla \boldsymbol{f}(\boldsymbol{X}))^{T} \boldsymbol{\lambda}=0 \\
& \boldsymbol{f}(\boldsymbol{X})+\boldsymbol{s}=0 \\
& \boldsymbol{L} \boldsymbol{S} \boldsymbol{e}-\mu \boldsymbol{e}=0
\end{aligned}
$$

These relations contain 11 variables $(\boldsymbol{X}, \boldsymbol{\lambda}, \boldsymbol{s})$ and 11 nonlinear equations. An approximate solution can be calculated when the value of $\mu$ is known.

Using this method, we can calculate the closest distance and the corresponding most critical time of the needle-insertion path. If we only want to rate the needleinsertion path, the variable $t$ should be set to a constant value when the needle tip has been inserted into the target. This value can be calculated from the needle-insertion length and speed rate of insertion.

\section{Simulation and Results}

Simulations were performed using a workstation with an AMD Opteron 248 processor, 4 GB of random access memory and running the Windows XP operating system.

\subsection{Implicit Functional Fitting of Surfaces Based on Sample Points of Related Structures during Planning}

The sample points on the surfaces of the related structures were obtained in the coordinate system of the robot by using the US probe and EM device [47], as shown in Fig. 7. Figure 8(a) shows the picture of needles and Figure $8(\mathrm{~b})$ shows the sample points of needle surface obtained by plastic probe [47]. Figure 9 (a) shows the 3D sketch of ribs in Solidworks software which is constructed by the sample points (Fig. 9(b)) obtained by the same method. Figure 10(a) shows the sketch of blood vessels in Ansys software which is constructed by the sample points 


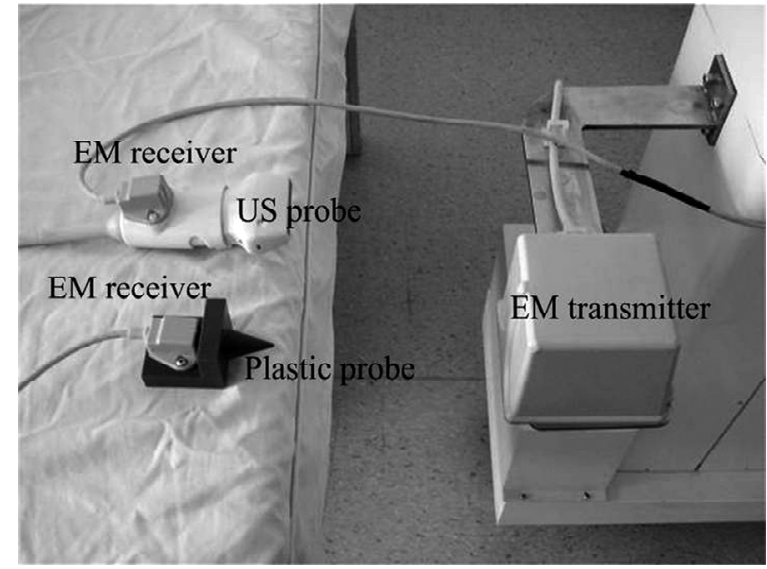

Figure 7. Sample points acquisition system.

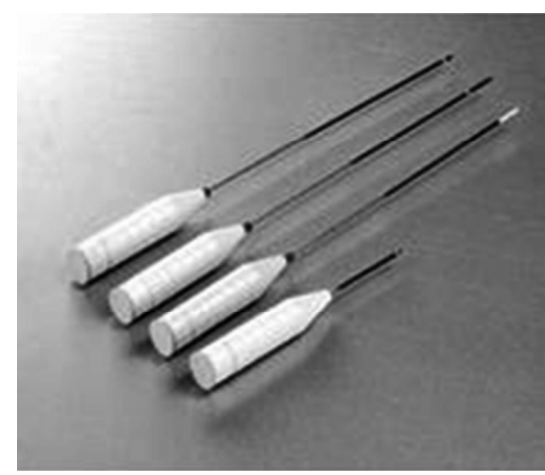

(a)

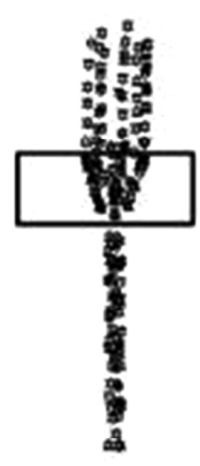

(b)
Figure 8. Needles (a) and its sample points of one needle (b).

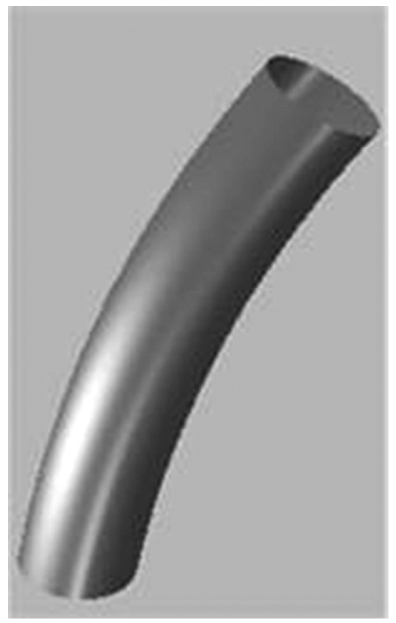

(a)

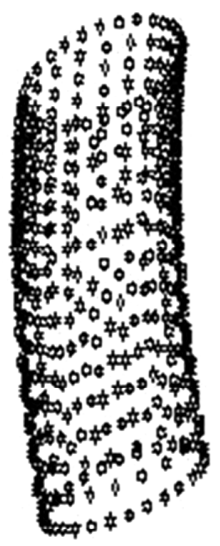

(b)
Figure 9. 3D sketch of ribs in Solidworks (a) and its sample points of the rib (b).

(Fig. 10(b)) obtained by edge extraction based on the 2D image scanning by the US probe. Figure 11 shows the sample points of abdominal epidermis surface also obtained by the plastic probe [47].

By using generalized characteristic vector fitting described in Section 3.5, the surface of the obstacles can be

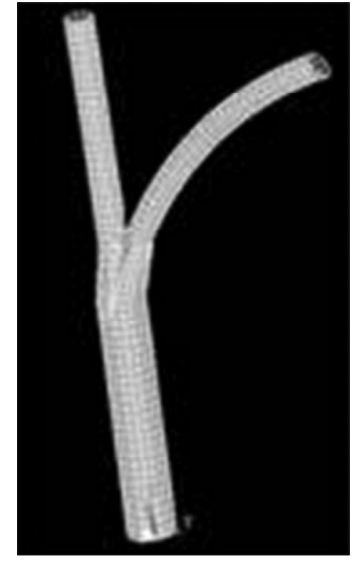

(a)

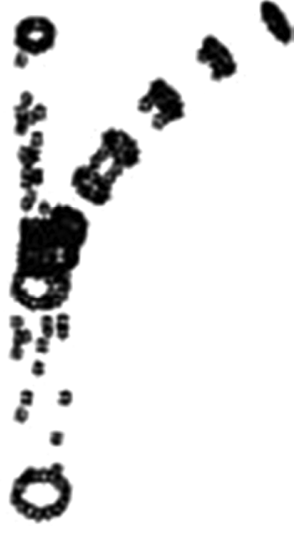

(b)
Figure 10. 3D sketch of blood vessels (a) and its sample points (b).

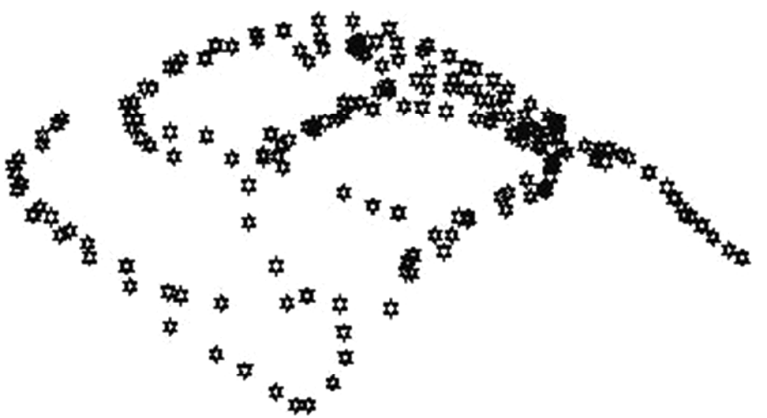

Figure 11. Sample points of abdominal epidermis surface.

fitted by implicit polynomials as shown in Fig. 12. Table 1 lists the fitting times and the errors. The fitting error is defined as the average of the shortest distances between each of the sample points and the fitted surface. The fits were obtained in a relatively short time, and the accuracy of the fits was good. Furthermore, the method does not require selection of an initial point.

Using this solution process, so long as a suitable set of sample points of the surface of the object is obtained, a corresponding implicit function describing that surface may also be obtained. When deformation of the liver is considered during surgical planning, we need to obtain only the sample points of the blood vessels following this deformation, and the surface can be fitted using the method. This method is also applicable to describe other objects where the topological structure is not complex. This step provides effective constraints for subsequent surgical planning. In other words, the implicit function describing the obstacle surface can be used to provide constrains to calculate the needle CFRW.

\subsection{Simulated Boundaries of the Needle CFRW}

Without loss of generality, a needle CFRW calculation was simulated using the obstacles (primary blood vessels and ribs) described in Section 4.1 as the obstacles. The simulation abdominal operation environment is shown in 


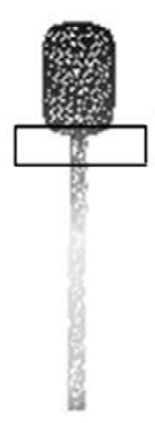

(a)

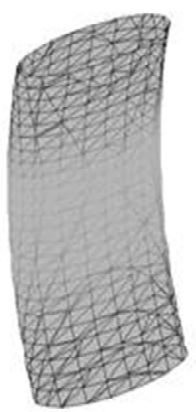

(b)

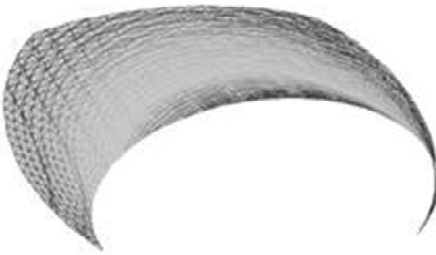

(c)

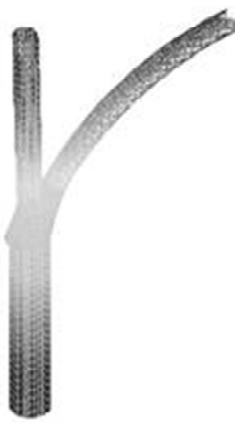

(d)

Figure 12. A schematic diagram showing the fitted surface of related structures: (a) the needle; (b) the rib; (c) abnormal surface; and (d) blood vessels. (We should point out that there is a simplification during the fitting of needle, because the part marked in Fig. 8(b) is useless for the collision detection.)

Table 1

Times Required to Achieve the Fits, together with the Associated Error

\begin{tabular}{|l|c|c|}
\hline Objects & Error $(\mathrm{mm})$ & Program Run Time (s) \\
\hline Needle & 0.188 & 0.205 \\
\hline Rib & $4.77 \mathrm{e}-7$ & 0.243 \\
\hline Abdominal surface & 0.218 & 0.151 \\
\hline Blood vessel & 0.375 & $2.71 \mathrm{e}-5$ \\
\hline
\end{tabular}

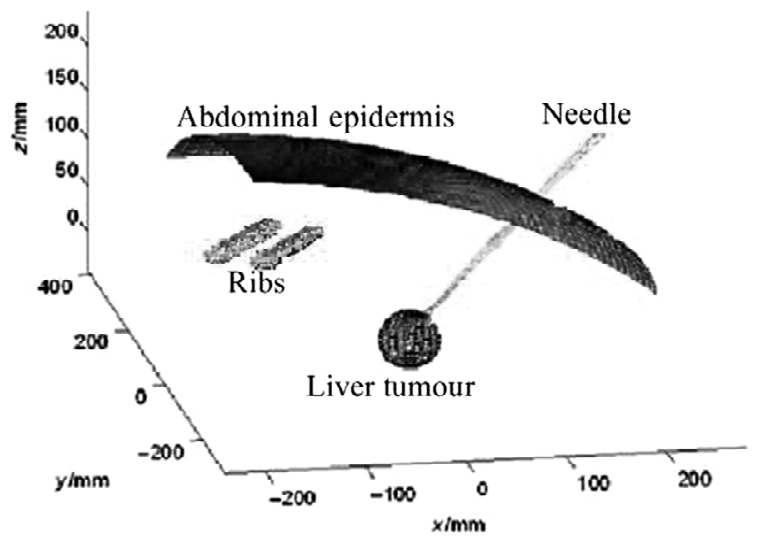

(a)

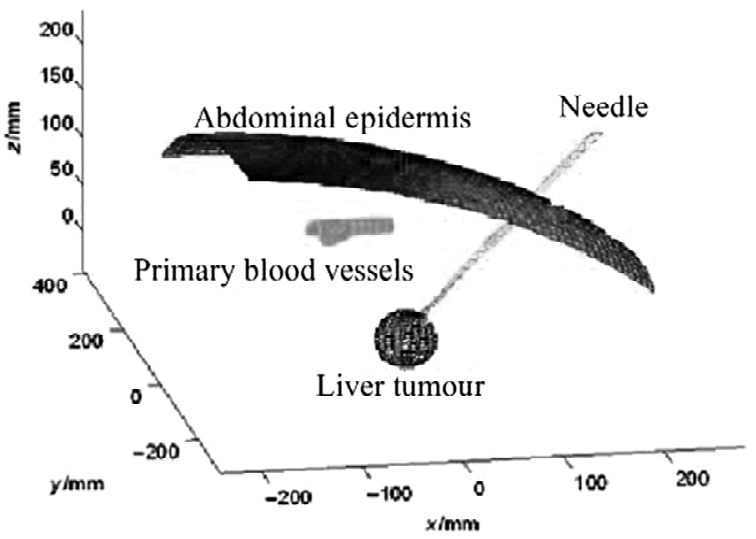

(c)
Fig. 13, where $O=[0,0,0]^{T} \mathrm{~mm}$ is the target point. We described the needle, ribs, blood vessels and abdominal epidermis by implicit functions. The simulation task is to calculate the needle CFRW with the ribs or blood vessels as obstacles and verify whether the insertion paths in the needle CFRW will be interfere with the obstacles.

Based on the method above, the 1D boundary of the needle CFRW in the $z_{h}$ plane with $z=h, h \in\left[0, h_{\max }\right]$ (according to our clinical experience, $h_{\max }=200 \mathrm{~mm}$ in our RALTCT system) can be described with (14). Then, the range of the angle of rotation $\theta$ was determined based on our RALTCT system, which is $0^{\circ}<\theta<45^{\circ}$.

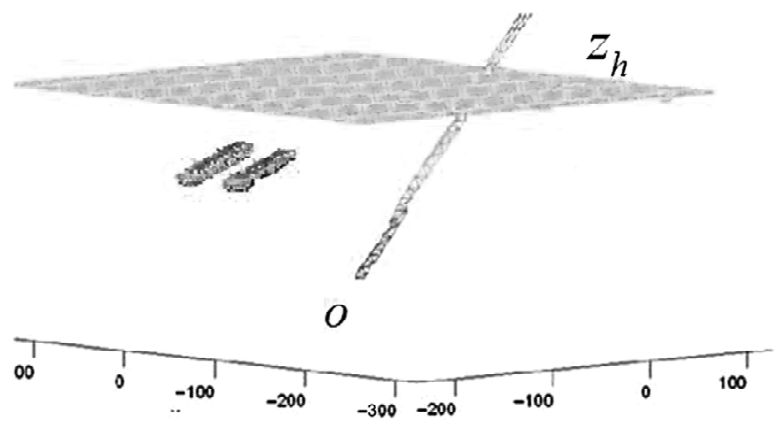

(b)

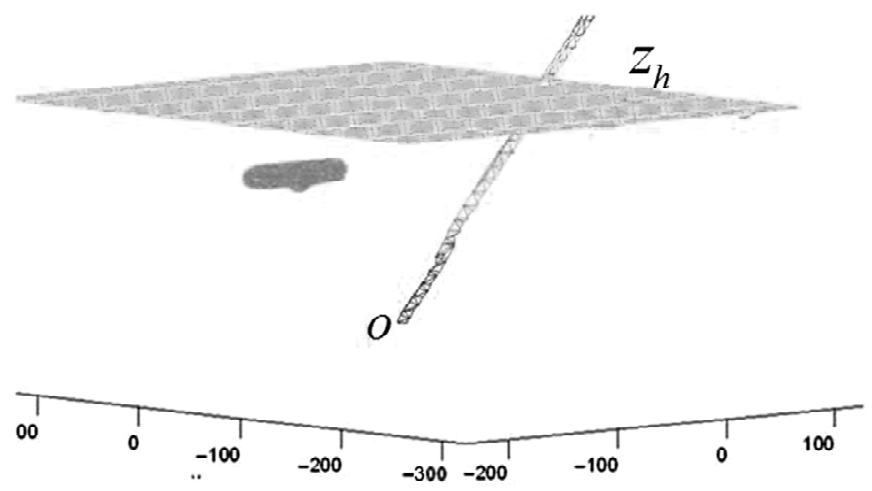

(d)

Figure 13. Schematic diagram of needle surgical planning environment: (a) simulation abdominal operation environment with ribs as obstacles; (b)needle and ribs; (c) simulation abdominal operation environment with blood vessels as obstacles; and (d) needle and blood vessels. 


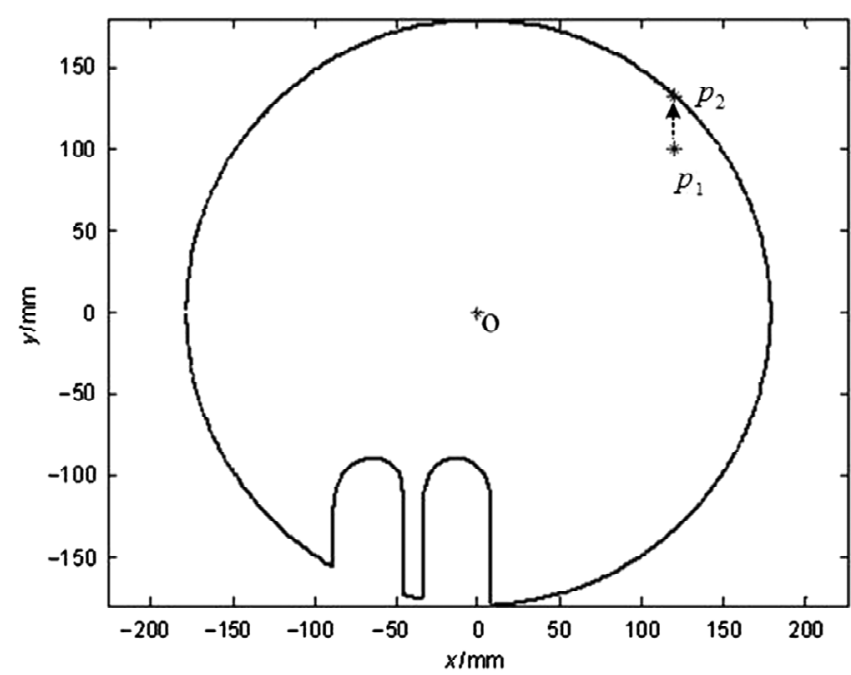

Figure 14. Boundaries of the simulated data for the ribs.

In an attempt to avoid the initial-value sensitivity of the parameterized nonlinear equations, the continuation method [48] was used to solve the boundary of the needle CFRW such that the 1D manifold in each $x y$ planar layer is obtained.

The procedure is as follows:

1. Select a point within the needle workspace and assign a search direction.

2. Move the point along the search direction until it reaches a boundary.

3. Trace the 1D manifold from that point on the boundary.

It has been shown that the trace in step (3) can repeatedly predict the following point along the tangent vector [48], and this can be used to correct the predicted point of the solution manifold, which is the solution of (14). The simulation software is developed to solve the boundary of the needle CFRW by using Visual $\mathrm{C}++6.0$ and visualize by MATLAB.

On the $x y$ plane with $z=160 \mathrm{~mm}$, we chose initial search point $\boldsymbol{P}_{1}=[121.6325,98.3184]^{T}$ within the workspace to solve (14). $\boldsymbol{P}_{2}=[121.6325,130.2372]^{T} \mathrm{~mm}$ is the point on the boundary obtained by searching from $\boldsymbol{P}_{1}$ along the $[0,1]^{T}$ direction. Setting $\gamma=1.5 \mathrm{~mm}$ and $\mu=0.1$, the 1D manifold was traced using the abovementioned continuation method. Figure 14 shows the boundary of the needle CFRW within the $x y$ plane at $z=160$ for the ribs.

Figure 15 shows the 3D boundaries of the needle CFRW within $x y$ planes of $z=150,160,170$ for the ribs. It appears that several groups of 1D manifolds in each $x y$ plane construct the 3D boundary.

As shown in Fig. 16, the lines are the needle-insertion trajectories, the entry points of which are on the boundaries. We calculated the closest distances between the lines and ribs, all of which are $1.5 \mathrm{~mm}$. These results are consistent with the settings above, such as $\gamma=1.5$. This also confirmed that the safe distance $\gamma$ could be adjusted according to the actual operation to control the closest distance between the needle-insertion trajectory and obstacles. Also, we can see that the needle-insertion trajectories in the needle CFRW will not interfere with obstacles.

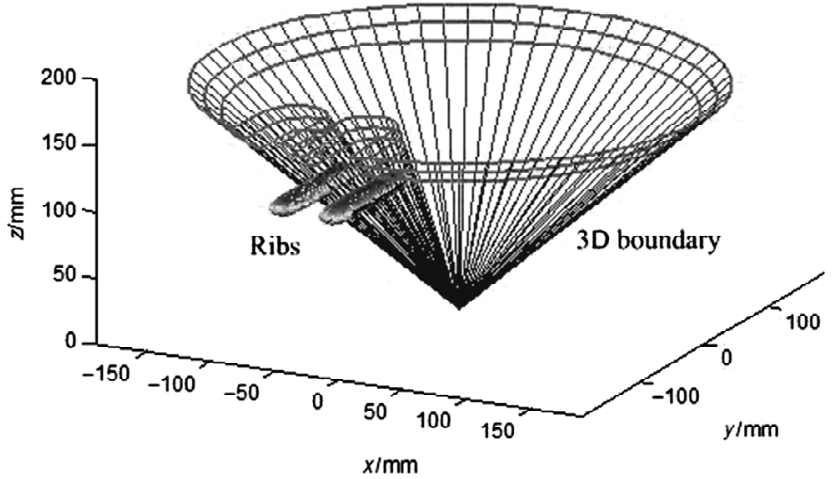

Figure 15. Three-dimensional-simulated boundaries of the ribs.

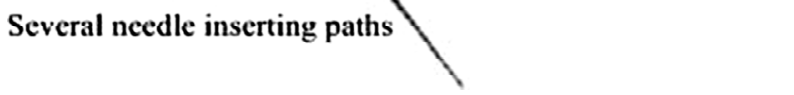

Figure 16. The local enlarged map.

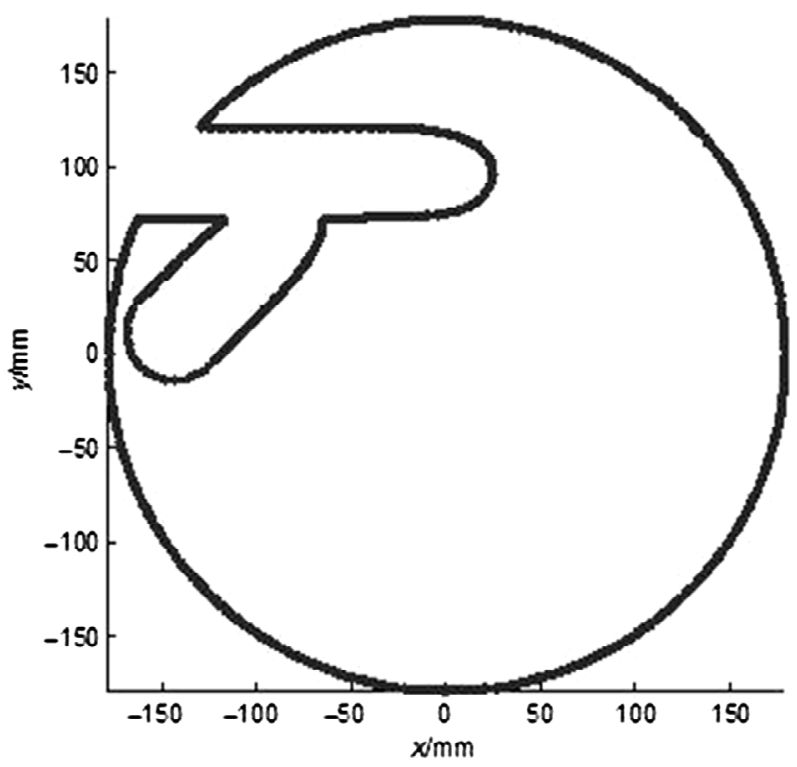

Figure 17. Simulated boundaries for primary blood vessels.

The same solution process was carried out with the primary blood vessels as the obstacles, Fig. 17 shows the boundary of the needle CFRW within the $x y$ plane at $z=160$ for the primary blood vessels, and Fig. 18 shows the $3 \mathrm{D}$ boundaries of the needle CFRW within the $x y$ plane at $z=150,160,170$ for the primary blood vessels.

Similarly, as shown in Fig. 19, the lines are the needle-insertion trajectories with the entry points on the boundaries. We calculated the closest distances between the lines and blood vessels, all of which are $1.5 \mathrm{~mm}$. The safe distances between the primary blood vessels and the 


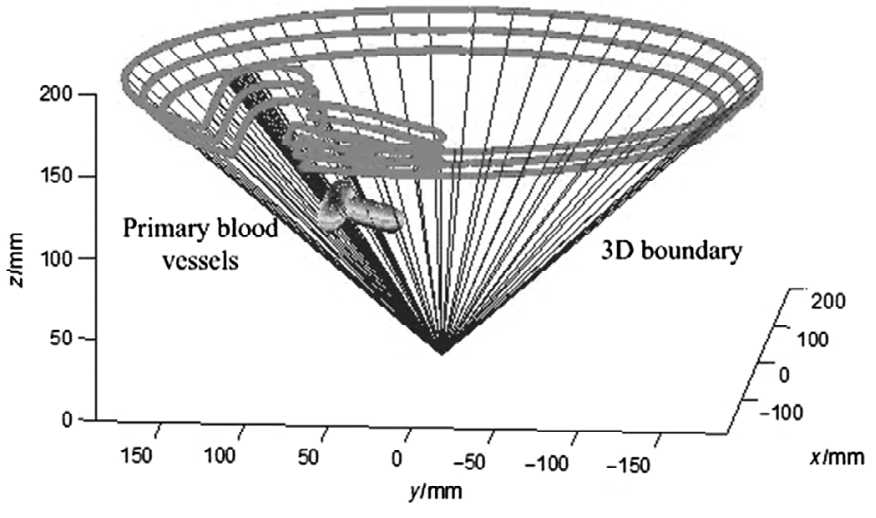

Figure 18. Three-dimensional-simulated boundaries for the blood vessels.

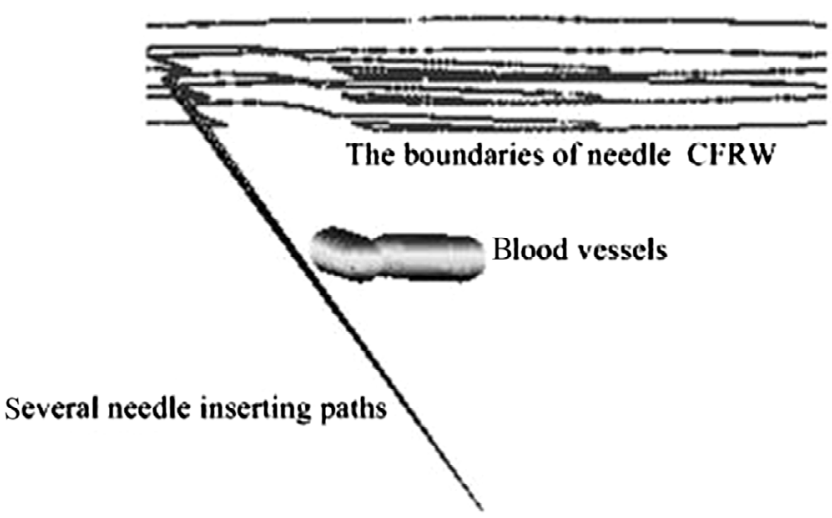

Figure 19. The local enlarged map.

needle-insertion paths indicate that we may expect no collisions, and the safe distance can be adjusted according to the operating conditions.

To sum up, the simulation results indicate that the needle-insertion trajectories which are in the corresponding needle CFRW will not damage ribs and blood vessels. Additionally, the simulation results indicate that the proposed method is applicable to different operation environments, as long as the obstacles can be represented by implicit functions. Thus, the deformation of the liver caused by needle insertion can be considered during planning. Based on the method in [47], the point cloud of obstacles and a new target can be obtained. According to the point cloud, the implicit function describing the obstacles can be calculated by a fitting algorithm in Section 3.5. The proposed method is also independent of obstacle size and position.

\subsection{Simulated Risk Analysis during the Surgery}

Once the CFRW of the needle has been obtained, the surgeon can select a feasible needle-insertion path in it. However, during surgery, the needle may pass close to the critical structures. Therefore, an intraoperative risk analysis which can obtain the closest distance from the needle-insertion path to the critical structures as well as the time parameters when the distance between the needle and the obstacles is closest can help the surgeon avoid some dangerous operation.

Figure 20 shows a needle-insertion path that the surgeon selects in the needle CFRW obtained in the last sec-

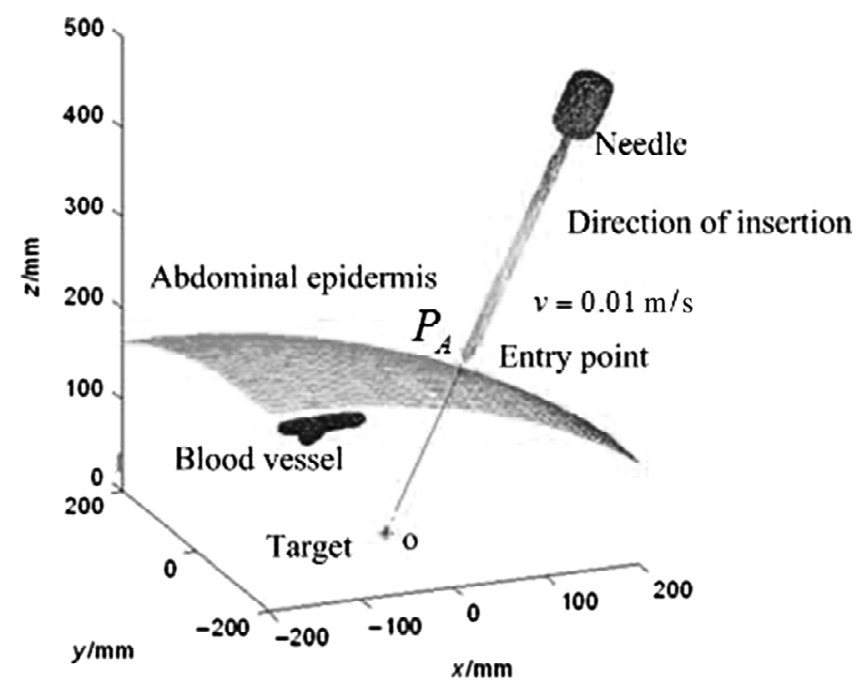

Figure 20. The risk analysis of needle-insertion path.

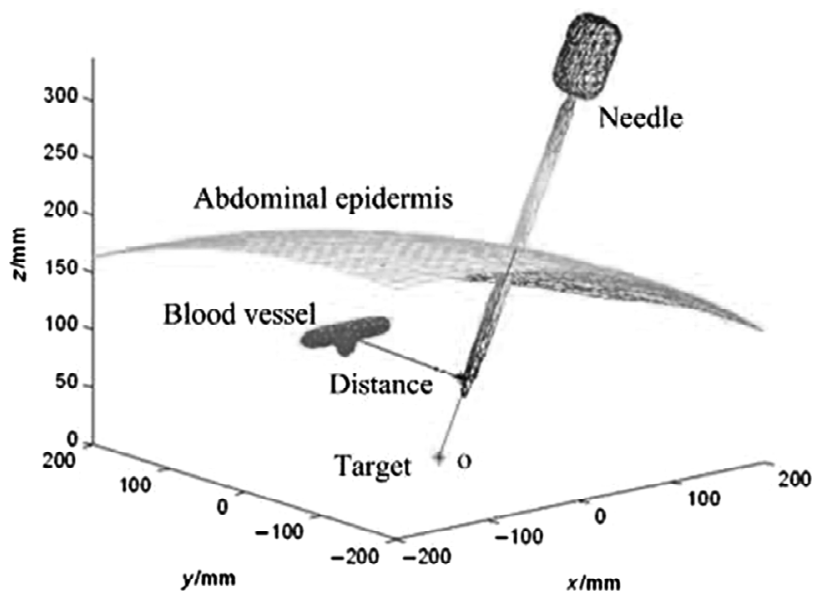

Figure 21. The shortest distance between the insertion needle and blood vessels and the corresponding needle pose of risk moment.

tion with blood vessels as the obstacles. Based on the risk analysis method, the closest distance between the selected path and the critical structures can be obtained, also the corresponding time can be solved.

The centre of each convex body (blood vessel and needle) is selected as the initial iteration point $\boldsymbol{X}_{0}$ in the algorithm, and based on the clinical conditions, we set $v=0.01 \mathrm{~m} / \mathrm{s}, \mu_{0}=0.2$. During each iteration, $\mu$ is multiplied by a constant $c=0.3$ to achieve convergence. For each value of $\mu$, the nonlinear equations are solved using the Newton method, where each step is determined using the trust region method [46]. The tolerance error was set to $10^{-5}$, and the closest points of the critical structures to the needle along the insertion path were calculated (Fig. 21). At the same time, the corresponding moment is also obtained. The results are shown in Table 2. The shortest distance between the needle-insertion path and blood vessel is $117 \mathrm{~mm}$ and the corresponding moment is $11.8 \mathrm{~s}$ after the beginning of insertion. The shorter the separation between the needle and the critical structures is, the more dangerous the needle-insertion path is. The surgeon may rate the paths according to the result of this 
Table 2

Result of Risk Analysis

\begin{tabular}{|l|c|c|c|}
\hline Object & $\begin{array}{c}\text { The Closest } \\
\text { Distance } \\
(\mathrm{mm})\end{array}$ & $\begin{array}{c}\text { The Corresponding } \\
\text { Moment } \\
(\mathrm{s})\end{array}$ & $\begin{array}{c}\text { Program } \\
\text { Run Time } \\
(\mathrm{s})\end{array}$ \\
\hline Distance & 117 & 11.8 & 0.893 \\
\hline
\end{tabular}

shortest distance calculation. To prove that the algorithm is fast, 100 entry points were randomly selected to solve the shortest distance between the needle-insertion path and critical structures. The program run time indicates that the frequency of the program is about $1.5 \mathrm{~Hz}$, this fully meet the real-time requirements of intraoperative surgical risk analysis.

In conclusion, the results of three simulation experiments indicate that: (1) an implicit function describing the surface of object in the surgery environment can be obtained by the proposed fitting method. Then the implicit function which has the enough fitting precision can be used to calculate the needle CFRW; (2) the needle-insertion path selected within the boundary of the needle CFRW will not interference with obstacles such as primary blood vessels, ribs and other important tissue. The boundary of the needle CFRW is an effective basis for the optimal needle-insertion path. We also show that this method can be applied to any obstacle, as long as the obstacle can be modelled as the third-order continuously differentiable implicit function. Therefore, the proposed method is applicable to various operating conditions. However, the insertion accuracy will be affected by the target motion, which is inevitable in the clinical surgery. As the insertion is monitored by 2D US imaging during the intraoperative stage in clinical surgery, the US probe was used to exert some pressure onto the patient abdomen, resulting in the deformation and motion of the liver. Also, the organ and lesion motion due to the respiratory effects will severely affect the needle placement accuracy and even cause the missing of the target in some cases [49]. Therefore, the target motion may be caused by the needle insertion, uneven pressure exerted by the US probe and the respiratory motion of the patient. Fortunately, the proposed method can be used to reduce needle placement error by target displacement compensation, as the displacement can be easily compensated into the proposed method once a reliable organ deformation model is available, which will be studied in our future work; and (3) during the surgery, the risk analysis can help surgeon assess the selected path and calculate the time parameters when the distance between the needle and the obstacles is closest. Also, the program run time fully meets the real-time requirements.

\section{Conclusion}

We have described a method of determining analytical expressions for the needle CFRW for surgical planning in abdominal surgery. The needle was modelled as an arm with a single ball-hinge joint, where the centre coincides with the location of the target. In this case, planning the needle-insertion path is simplified to a search of entry points because the target is fixed during the procedure. An analytical expression for the boundary of the needle CFRW was obtained using the parameterized nonlinear equations. The surgeon can select the entry point from the needle CFRW. A risk analysis method was developed to calculate the closest separation between the needle-insertion path and the critical structures. Using this method, the paths can be rated and the surgeon can monitor the insertion path during surgery using US images.

Simulation results indicate that an entry point can be chosen within the boundary of the needle CFRW that guarantees that the corresponding needle-insertion path will not interfere with primary blood vessels, ribs, or other critical organs or tissues. If mathematical descriptions of the obstacles are given, the preoperative planning method can provide a feasible solution set of needle-insertion paths. Based on our method, the clinical constraints and rating factors - such as the safety margin around target constraints, tangency constraints, needle length constraints, the distances to critical structures and trajectory length - are expressed as parameters in the nonlinear equations that describe the needle CFRW under the prerequisites, which include segmentation of the important organs and the implicit expression of these organs' surfaces. The needle-insertion path method described here is suitable for obstacles of various size, shape and position. Additionally, the method is independent of the mesh representation of related structures and the resolution of the screen used. Therefore, it is more accurate. As part of future work, we plan to consider deformation of soft tissue, constraints describing the motion of the robot movement, the optimal location of the base of the robot and automatic optimal needle-insertion trajectory selection, which can be obtained within the needle CFRW according to designed optimization criteria such as the shortest distance between the entry point and the target point. All of these are expected to improve the accuracy of the method.

\section{Acknowledgement}

Most of the work presented in this paper is conducted by the Digital Design \& Manufacturing Laboratory at Beijing Institute of Technology, Robot \& Automation Lab (RAL) at Tsinghua University and the Chinese PLA General Hospital. Financial support is mainly provided by National Natural Science Foundation of China (NSFC) under Grant No. 51305031 and National Defense Fundamental Research Foundation, China (No. JCKY2016204B201).

\section{References}

[1] A. Jacobs, Radiofrequency ablation for liver cancer, Radiologic Technology, 86(6), 2015, 645-664.

[2] J. Xu, Z.Z. Jia, Z.J. Song, X.D. Yang, et al., Three-dimensional ultrasound image-guided robotic system for accurate microwave coagulation of malignant liver tumors, International Journal of Medical Robotics and Computer Assisted Surgery, 6(3), 2010, 256-268.

[3] M. Peterhans, A. vom Berg, B. Dagon, D. Inderbitzin, et al., A navigation system for open liver surgery: Design, workflow and first clinical applications, International Journal of Medical Robotics and Computer Assisted Surgery, 7(1), 2011, 7-16. 
[4] N. Shevchenko, B. Seidl, J. Schwaiger, M. Markert, et al., MiMed Liver: A planning system for liver surgery, 32nd Annu. Int. Conf. IEEE EMBS, Buenos Aires, 2010, 1882-1885.

[5] J. Schwaiger, M. Markert, B. Seidl, N. Shevchenko, et al., Risk analysis for intraoperative liver surgery, 32nd Annu. Int. Conf. IEEE EMBS, Buenos Aires, 2010, 410-413.

[6] Y.W. Chen, M. Kaibori, T. Shindo, K. Miyawaki, et al., Computer-aided liver surgical planning system using CT volumes, 32nd Annu. Int. Conf. IEEE EMBS, Osaka, 2013, $2360-2363$.

[7] Y. Zhong, B. Shirinzadeh, J. Smith, Soft tissue deformation with neural dynamics for surgery simulation, International Journal of Robotics \& A Atomation, 22(1), 2007, 1-9.

[8] V.M. Banz, P.C. Müller, P. Tinguely, D. Inderbitzin, et al., Intraoperative image-guided navigation system: Development and applicability in 65 patients undergoing liver surgery, Langenbeck's Archives of Surgery, 401(4), 2016, 495-502.

[9] B. Abdullah, C. Yeong, K. Goh, B. Yoong, et al., Roboticassisted thermal ablation of liver tumours, European Radiology, 25(1), 2015, 246-257.

[10] M.A. Scherer and D.A. Geller, New preoperative images, surgical planning, and navigation, in Y. Fong, P. Giulianotti, J. Lewis, B. K. Groot, and T. Reiner (eds.), Imaging and Visualization in The Modern Operating Room, (New York: Springer-Verlag, 2015), 342-366.

[11] B. Chebbi, D. Lazaroff, and P.X. Liu, A collaborative virtual haptic environment for surgical training and tele-mentoring, International Journal of Robotics \& Automation, 22(1), 2007, 69-78.

[12] S. Cristina, G. Gorka, G. Purificacion, G. Tomas, et al., Personalized surgical planning to support interventions and training of surgeons, Clinical Image-Based Procedures, Planning to Intervention Lecture Notes in Computer Science, 7761, 2013, 83-90.

[13] 3D Slicer. Available at: http://www.slicer.org.

[14] Available at: neromate.http://www.renishaw.com/en/neuro mate-the-no-1-image-guided-neurosurgical-robot-10712.

[15] M. Hayashibe, N. Suzuki, M. Hashizume, Y. Kakeji, et al., Preoperative planning system for surgical robotics setup with kinematics and haptics, International Journal of Medical Robotics and Computer Assisted Surgery, 1(2), 2005, 76-85.

[16] L.B. Rosenberg, Virtual fixtures: Perceptual tools for telerobotic manipulation, IEEE Virtual Reality Annual Int. Symp., Seattle, WA, USA, 1993, 76-82.

[17] J.J. Abbott, P. Marayong, and A.M. Okamura, Haptic virtual fixtures for robot-assisted manipulation, Springer Tracts in Advanced Robotics, 28, 2007, 49-64.

[18] T. Xia, A. Kapoor, P. Kazanzides, and R. Taylor, A constrained optimization approach to virtual fixtures for multirobot collaborative teleoperation, 2011 IEEE/RSJ Int. Conf. Intelligent Robots and Systems, Francisco CA, 2011, 639-644.

[19] S. Park, R.D. Howe, and D.F. Torchiana, Virtual fixtures for robotic cardiac surgery, Lecture Notes in Computer Science, 2208, 2001, 1419-1420.

[20] A. Kapoor, M. Li, and R.H. Taylor, Constrained control for surgical assistant robots, IEEE Int. Conf. Robotics and Automation, Orlando, 2006, 231-236.

[21] B. Duan, R. Wen, C. Chng, W. Wang, et al., Image-guided robotic system for radiofrequency ablation of large liver tumor with single incision, 12th Int. Conf. Ubiquitous Robots and Ambient Intelligence, Goyang, 2015, 284-289.

[22] H. Ren, E. Campos-Nanez, Z. Yaniv, F. Banovac, et al., Treatment planning and image guidance for radiofrequency ablation of large tumors, IEEE Journal of Biomedical and Health Informatics, 18(3), 2014, 920-928.

[23] L. Adhami and E. Coste-Maniere, Optimal planning for minimally invasive surgical robots, IEEE Transactions on Robotics, 19(5), 2003, 854-863.

[24] L. Adhami, E. Coste-Maniere, and J.D. Boissonnat, Planning and simulation of robotically assisted minimal invasive surgery, Medical Image Computing and Computer-Assisted Intervention, 1935, 2000, 624-633.

[25] M. Vaillant, C. Davatzikos, R.H. Taylor, and R.N. Bryan, A path-planning algorithm for image-guided neurosurgery, Lecture Notes in Computer Science, 1205, 1997, 467-476.
[26] R.R. Shamir, I. Tamir, E. Dabool, L. Joskowicz, et al., A method for planning safe trajectories in image-guided keyhole neurosurgery, Medical Image Computing and ComputerAssisted Intervention, 13(3), 2010, 457-464.

[27] C. Essert, C. Haegelen, F. Lalys, A. Abadie, et al., Automatic computation of electrode trajectories for deep brain stimulation: A hybrid symbolic and numerical approach, International Journal of Computer Assisted Radiology and Surgery, 7(4), 2011, 517-532.

[28] C. Baegert, C. Villard, P. Schreck, and L. Soler, Multi-criteria trajectory planning for hepatic radiofrequency ablation, Medical Image Computing and Computer-Assisted Intervention, 4792, 2007, 676-684.

[29] C. Baegert, C. Villard, P. Schreck, and L. Soler, Precise determination of regions of interest for hepatic RFA planning, Studies in Health Technology and Informatics, 125, 2007, 31-36.

[30] C. Villard, C. Baegert, P. Schreck, L. Soler, et al., Optimal trajectories computation within regions of interest for hepatic RFA planning, Medical Image Computing and Computer-Assisted Intervention, 8, 2005, 49-56.

[31] A. Seitel, M. Engel, C.M. Sommer, B.A. Radeleff, et al., Computer-assisted trajectory planning for percutaneous needle insertions, Medical Physics, 38(6), 2011, 3246-3259.

[32] C. Schumann, J. Bieberstein, C. Trumm, D. Schmidt, et al., Fast automatic path proposal computation for hepatic needle placement, Medical Imaging 2010: Visualization, ImageGuided Procedures, and Modeling, 7625, 2010, 76251J$76251 \mathrm{~J}-10$.

[33] C. Schumann, C. Rieder, S. Haase, K. Terchert, et al., Interactive multi-criteria planning for radiofrequency ablation, International Journal of Computer Assisted Radiology and Surgery, 10(6), 2015, 879-889.

[34] A. Kapoora, M. Li, and B. Wood, Mixed variable optimization for radio frequency ablation planning, Medical Imaging 2011: Visualization, Image-Guided Procedures, and Modeling, 7964, 2011, 796420-1-796420-7.

[35] C.C. Chen, M.I. Miga, and R.L. Galloway Jr, Optimizing electrode placement using finite-element models in radiofrequency ablation treatment planning, IEEE Transactions on Biomedical Engineering, 56(2), 2009, 237-245.

[36] S. Haase, P. Süss, J. Schwientek, K. Teichert, et al., Radiofrequency ablation planning: An application of semi-infinite modelling techniques, European Journal of Operational Research, 218(3), 2012, 856-864.

[37] A. Jaberzadeh and C. Essert, Pre-operative planning of multiple probes in three dimensions for liver cryosurgery: Comparison of different optimization methods, Mathematical Methods in the Applied Sciences, 39(16), 2015, 4764-4772.

[38] X. Yang, H. Wang, C. Zhang, and K. Chen, A method for mapping the boundaries of collision-free reachable workspaces, Mechanism and Machine Theory, 45(7), 2010, 1024-1033.

[39] N. Chakraborty, J. Peng, S. Akella, and J.E. Mitchell, Proximity queries between convex objects: An interior point approach for implicit surfaces, IEEE Transactions on Robotics, 24(1), 2008, 211-220.

[40] A.J. Hanson, Hyperquadrics: Smoothly deformable shapes with convex polyhedral bounds, Computer Vision, Graphics, and Image Processing, 44(2), 1988, 191-210.

[41] S. Boyd and L. Vandenberghe, Convex optimization (Chicago: University of Chicago Press, 2004).

[42] G. Taubin, Nonplanar curve and surface estimation in 3-space, IEEE Int. Conf. Robotics and Automation, Philadelphia, PA, USA, 1988, 644-645.

[43] D. Keren and C. Gotsman, Fitting curves and surfaces with constrained implicit polynomials, IEEE Transactions on Pattern Analysis and Machine Intelligence, 21(1), 1999, 31-41.

[44] J.E. Dennis and R.B. Jr Schnabel, Numerical methods for unconstrained optimization and nonlinear equations, (Eaglewood Cliffs: Prentice-Hall, 1983).

[45] G. Taubin, F. Cukierman, S. Sullivan, J. Ponce, et al., Parametrizing and fitting bounded algebraic curves and surfaces, IEEE Computer Society Conference on Computer Vision and Pattern Recognition, Champaign, USA, 1992, 103-108.

[46] R. Byrd, J. Nocedal, and R.A. Waltz, An integrated package for nonlinear optimization: Large scale nonlinear optimization (New York: Springer-Verlag, 2006). 
[47] S.L. Liu, Research on multi-needle surgical planning of robotassisted microwave coagulation in liver cancer therapy, Doctoral Diss., Tsinghua University, Beijing, China, 2012 (in Chinese).

[48] W.C. Rheinboldt, Numerical analysis of parameterized nonlinear equations (New York: John Wiley \& Sons, 1986).

[49] E.M. Boctor, M.A. Choti, E.C. Burdette, and R.J. Webster III, Three-dimensional ultrasound-guided robotic needle placement: An experimental evaluation, The International Journal of Medical Robotics and Computer Assisted Surgery, 4(2), 2008, 180-191.

\section{Biographies}

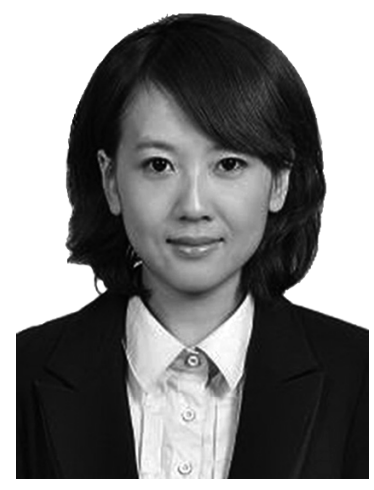

Shaoli Liu received the B.S. degree in mechanical engineering from Beijing Institute of Technology, Beijing, China, in 2007, and Ph.D. degree in mechanical engineering from Tsinghua University, Beijing, China, in 2012. Since 2012, she has been working at the School of Mechanical Engineering, Beijing Institute of Technology, Beijing, China. Her research is in the areas of medical robot, machine vision and on-line detection. She is interested in the image processing and 3D reconstruction for machine vision-based detection. Specifically, she is studying the calibration of cameras, point clouds processing, 3D reconstruction for precision detection.

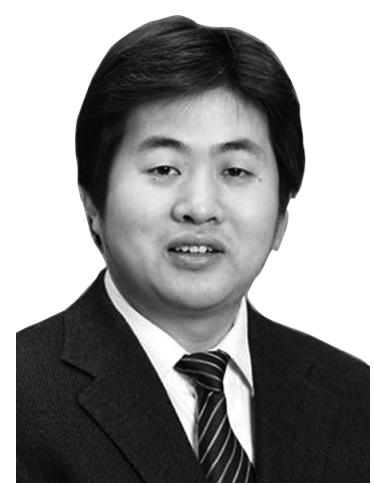

Jianhu L Liu received the B.S. and M.S. degrees in mechanical engineering from Chongqing University, Chongqing, China, in 1999 and 2002, and Ph.D. degree in mechanical engineering from Beijing Institute of Technology, Beijing, China, in 2005. Since 2005, he has been working at the School of Mechanical Engineering, Beijing Institute of Technology. His research interests include digital assembly, bolted joints and digital inspection. Specifically, he focuses on modelling and simulation of digital assembly process for complex mechanical and electrical products; digital layout design, assembly and inspection of pipes and cables; tightening process, loosening mechanism and anti-loosening measures of bolts.

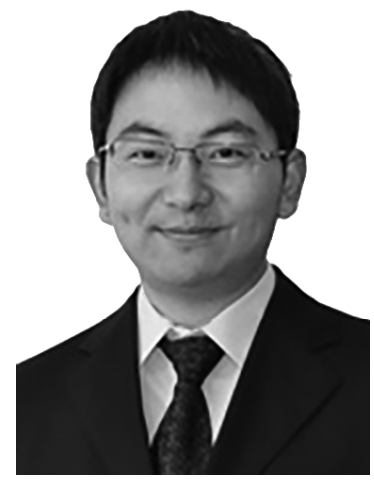

Jing $X u$ received the B.S. degree in mechanical engineering from Harbin Institute of Technology, Harbin, China, in 2003, and the Ph.D. degree in mechanical engineering from Tsinghua University, Beijing, China, in 2008. He was a Postdoctoral Researcher in the Department of Electrical and Computer Engineering, Michigan State University, East Lansing. He is currently an Associate
Professor in the Department of Mechanical Engineering, Tsinghua University, Beijing, China. His research interests include vision-guided manufacturing, image processing and intelligent robotics.

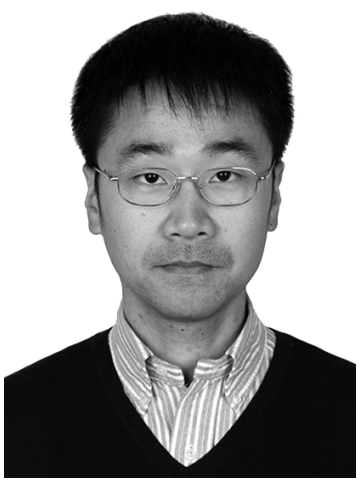

Xiaoyu Ding received the B.S. and M.S. degree in mechanical engineering from Tsinghua University, Beijing, China, in 2006 and 2009, and Ph.D. degree in mechanical engineering from Georgia Institute of Technology, Atlanta, USA, in 2014. Since 2014, he has been working at the School of Mechanical Engineering, Beijing Institute of Technology. His research is focused on exploring computer-aided design and virtual assembly technologies to inform design decisions and enhance or predict the performance of products. In addition, he develops innovative computer-aided tolerancing methods that allow for accurate and efficient prediction of stress-induced assembly errors.

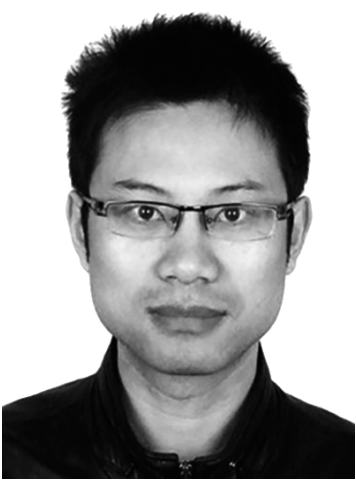

Tong $L u$ received the B.S. degree in Clinical Medicine from The Fourth Military Medical University, Xi'an, China, in 2000, and the Ph.D. degree in Clinical Medicine from Medical School of Chinese PLA, Beijing, China, in 2010. He is currently the Chairman of the Board in Beijing Wei Zhuo Zhi Yuan Medical Science and Technology Development Co., LLC, Beijing, China. His research interests include medical image $3 \mathrm{~d}$ visualization, mixed reality, and surgical navigation.

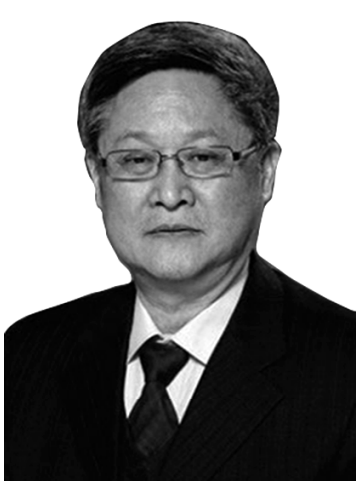

Ken Chen received the B.S. degree, M.S. degree and Ph.D. degree in mechanical engineering from Zhejiang University, Hangzhou, China, in 1981, 1984 and 1987 separately. He was the associate professor at the School of Mechanical Engineering, Sichuan University from 1988 to 1991, the visiting professor at the University of Illinois from 1991 to 1992, the post doctor at Purdue University from 1993 to 1995. Since 1995, he has been working at Tsinghua University. He is currently a professor in the Department of Mechanical Engineering, Tsinghua University, Beijing, China. His research interests include robotics and manufacturing automation. 